\begin{tabular}{|c|l|}
\hline Title & Electron self-energy from quantum charge fluctuations in the layered t-J model with long-range Coulomb interaction \\
\hline Author(s) & Yamase, Hiroyuki; Bejas, Matias; Greco, A ndres \\
\hline Citation & $\begin{array}{l}\text { Physical Review B, 104(4), 045141 } \\
\text { https://doi.org/10.1103/PhysRevB.104.045141 }\end{array}$ \\
\hline Issue Date & 2021-07-26 \\
\hline Doc URL & http://hdl.handle.net/2115/82622 \\
\hline Rights & ○2021 A merican Physical Society \\
\hline Type & article \\
\hline File Information & PhysRevB.104_4_045141.pdf \\
\hline
\end{tabular}

Instructions for use 


\title{
Electron self-energy from quantum charge fluctuations in the layered $t-J$ model with long-range Coulomb interaction
}

\author{
Hiroyuki Yamase $\odot,{ }^{1,2}$ Matías Bejas $\odot,{ }^{3}$ and Andrés Greco $\odot^{3}$ \\ ${ }^{1}$ International Center of Materials Nanoarchitectonics, National Institute for Materials Science, Tsukuba 305-0047, Japan \\ ${ }^{2}$ Department of Condensed Matter Physics, Graduate School of Science, Hokkaido University, Sapporo 060-0810, Japan \\ ${ }^{3}$ Facultad de Ciencias Exactas, Ingeniería y Agrimensura and Instituto de Física Rosario (UNR-CONICET), \\ Av. Pellegrini 250, 2000 Rosario, Argentina
}

(Received 31 October 2020; accepted 7 July 2021; published 26 July 2021)

\begin{abstract}
Employing a large- $N$ scheme of the layered $t-J$ model with the long-range Coulomb interaction, which captures the fine details of the charge excitation spectra recently observed in cuprate superconductors, we explore the role of charge fluctuations on the electron self-energy. We fix the temperature at zero and focus on quantum charge fluctuations. We find a pronounced asymmetry of the imaginary part of the self-energy $\operatorname{Im} \Sigma(\mathbf{k}, \omega)$ with respect to $\omega=0$, which is driven by strong electron correlation effects. The quasiparticle weight is reduced dramatically, which occurs almost isotropically along the Fermi surface. Concomitantly, an incoherent band and a sharp side band are generated and acquire sizable spectral weight. All these features are driven by the usual on-site charge fluctuations, which are realized in a rather high-energy region and yield plasmon excitations. On the other hand, the low-energy region with the scale of the superexchange interaction $J$ is dominated by bond-charge fluctuations. Surprisingly, compared with the effect of on-site charge fluctuations, their effect on the electron self-energy is much weaker, even if the system approaches close to bond-charge instabilities. Furthermore, quantum charge dynamics does not produce a clear kink nor a pseudogap in the electron dispersion.
\end{abstract}

DOI: 10.1103/PhysRevB.104.045141

\section{INTRODUCTION}

High-temperature cuprate superconductors are realized upon charge-carrier doping into antiferromagnetic Mott insulators. Many studies have highlighted the magnetic properties. In particular, the effect of spin fluctuations has been a major subject to explore the cuprate phenomenology such as hightemperature superconductivity, the anomalous metallic state including the enigmatic pseudogap, and others [1]. Needless to say, the charge dynamics has also been widely recognized as an important subject. However, much was not known about the charge dynamics in momentum and energy space. Recently, advanced x-ray scattering techniques [2-8] changed this situation.

For electron-doped cuprates (e-cuprates), charge excitations were reported in both a low-energy region $[6,9]$ with a scale of the superexchange interaction $J(\approx 150 \mathrm{meV})$ and a high-energy region $[2,7,8,10-12]$ with a scale larger than $J$. These features were captured in the layered $t-J$ model with the long-range Coulomb interaction as a dual structure of the charge excitations [13]. The low-energy charge excitations originate mainly from the bond-charge fluctuations $[14,15]$ and the high-energy ones from acousticlike plasmons [16-18].

For hole-doped cuprates (h-cuprates), mainly low-energy charge fluctuations were reported [3-5] and their origin is under debate despite intensive theoretical studies [19-26]. The origin of high-energy charge excitations in h-cuprates [27] is also highly controversial. High-energy excitations can be (i) specific to e-cuprates [7,10], (ii) present as a broad peak of the particle-hole excitation spectrum [27], (iii) present as plasmons similar to the e-cuprates $[11,12,17,28,29]$, and (iv) related to strange metal physics [30,31], not to plasmons.

Cuprate superconductors are strongly correlated electron systems and the bare hopping integral $t$, whose energy scale is usually $\mathrm{eV}$, is renormalized to be in the scale of $J$, leading to a relatively narrow band width. In this case, a coupling to bosonic excitations such as charge fluctuations revealed recently is expected to generate anomalous features in the electron self-energy.

However, there are only a few studies along that motivation. Among others, Ref. [32] studied the effect of the optical plasmon on the electron dispersion in a phenomenological framework. Reference [33] discussed the effect of incoherent hole motion to explain the so-called high-energy kink of the electron dispersion observed by angle-resolved photoemission spectroscopy (ARPES) around $-(0.3 \sim 1) \mathrm{eV}$ (Refs. [34-39]). We still only have sparse insights into the effect of charge fluctuations on the one-particle properties. Hence, it is very interesting to apply the large- $N$ theory, which captures fine details of charge excitation spectra observed by experiments, to the study of the electron self-energy and to perform a comprehensive analysis of the one-particle properties of electrons in a microscopic model. Such a study will potentially open a path to study the charge dynamics in cuprates also from one-particle properties, which may accelerate our understanding of the cuprate physics.

In this paper, we employ the layered $t-J$ model with the long-range Coulomb interaction in a large- $N$ scheme and 
study the electron self-energy from charge fluctuations. This is a challenging study because we have to go beyond leading order in a large- $N$ expansion. We fix the temperature at zero and focus on quantum charge fluctuations. We shall clarify how the quantum charge dynamics renormalizes the quasiparticle dispersion and generates bands for both e- and h-cuprates, including a possibility of kinks and a pseudogap in the electron dispersion. Since the charge dynamics consists of on-site charge and bond-charge fluctuations, we sharply distinguish these two features in the present analysis.

The present paper is organized as follows. After providing a brief summary of the formalism in Sec. II, we present results in Sec. III. Starting from a summary of the typical charge excitation spectrum, we show the self-energy effect from on-site charge fluctuations, which describe plasmons, and then clarify the effect of bond-charge fluctuations. The obtained results are discussed from a view of both theoretical and experimental perspectives in Sec. IV. Concluding remarks are given in Sec. V. Appendices consist of two parts: a full description of the formalism and additional results for e-cuprates.

\section{MODEL AND FORMALISM}

As a microscopic model of cuprate superconductors, we study the $t-J$ model on a square lattice by including interlayer hopping and the long-range Coulomb interaction. The Hamiltonian is given by

$$
\begin{aligned}
H= & -\sum_{i, j, \sigma} t_{i j} \tilde{c}_{i \sigma}^{\dagger} \tilde{c}_{j \sigma}+\sum_{\langle i, j\rangle} J_{i j}\left(\vec{S}_{i} \cdot \vec{S}_{j}-\frac{1}{4} n_{i} n_{j}\right) \\
& +\frac{1}{2} \sum_{i, j} V_{i j} n_{i} n_{j},
\end{aligned}
$$

where $\tilde{c}_{i \sigma}^{\dagger}\left(\tilde{c}_{i \sigma}\right)$ are the creation (annihilation) operators of electrons with spin $\sigma(=\uparrow, \downarrow)$ in the Fock space without double occupancy at any site, $n_{i}=\sum_{\sigma} \tilde{c}_{i \sigma}^{\dagger} \tilde{c}_{i \sigma}$ is the electron density operator, $\vec{S}_{i}$ is the spin operator, and the sites $i$ and $j$ run over a three-dimensional lattice. The hopping $t_{i j}$ takes the value $t\left(t^{\prime}\right)$ between the first- (second-) nearest-neighbor sites on the square lattice and is scaled by $t_{z}$ between the layers; see Eqs. (3) and (4) for the explicit form of the electron dispersion. $\langle i, j\rangle$ denotes the nearest-neighbor sites and the exchange interaction $J_{i j}=J$ is considered only inside the plane. We neglect the exchange term between the planes, which is much smaller than $J$ (Ref. [40]). $V_{i j}$ is the long-range Coulomb interaction on the three-dimensional lattice and its functional form is given in momentum space later [Eq. (9)]. While cuprates are essentially two-dimensional systems, it is crucial to employ the three-dimensional model, namely, the layered model, because the long-range Coulomb interaction leads to a sizable momentum dependence of plasmons along the $q_{z}$ direction as was shown in classical papers [41-43].

It is highly nontrivial to treat the $t-J$ model Eq. (1) because of the local constraint prohibiting the double occupancy at any site. Here we employ a large- $N$ technique in a path integral representation in terms of the Hubbard operators [44]. In the large- $N$ scheme, the number of spin components is extended from 2 to $N$ and physical quantities are computed by counting the power of $1 / N$ systematically. One of the advantages of this method is that it treats all possible charge excitations on an equal footing $[19,45]$, which makes this method potentially interesting in light of the $\mathrm{x}$-ray experiments revealing excitation spectra in the pure charge channel [2-12,27-29].

The computation of the electron self-energy requires extended calculations in a large- $N$ framework beyond leading order theory [46-48]. Since we would like to highlight the effect of charge fluctuations on the self-energy in this paper, we present the most essential part of the formalism here, leaving its full description to Appendix A. In short, we first obtain the electron dispersion at leading order and compute charge fluctuations at the order of $1 / N$. We then calculate their contributions to the electron self-energy at the same order.

At leading order, the electron dispersion $\varepsilon_{\mathbf{k}}$ is obtained as

$$
\varepsilon_{\mathbf{k}}=\varepsilon_{\mathbf{k}}^{\|}+\varepsilon_{\mathbf{k}}^{\perp},
$$

where the in-plane dispersion $\varepsilon_{\mathbf{k}}^{\|}$and the out-of-plane disper$\operatorname{sion} \varepsilon_{\mathbf{k}}^{\perp}$ are given, respectively, by

$$
\begin{gathered}
\varepsilon_{\mathbf{k}}^{\|}=-2\left(t \frac{\delta}{2}+\Delta\right)\left(\cos k_{x}+\cos k_{y}\right)-4 t^{\prime} \frac{\delta}{2} \cos k_{x} \cos k_{y}-\mu \\
\varepsilon_{\mathbf{k}}^{\perp}=-2 t_{z} \frac{\delta}{2}\left(\cos k_{x}-\cos k_{y}\right)^{2} \cos k_{z}
\end{gathered}
$$

Here we measure the in-plane momenta $k_{x}$ and $k_{y}$ and the outof-plane momentum $k_{z}$ in units of $a^{-1}$ and $d^{-1}$, respectively; $a(d)$ is the lattice constant in the plane (distance between the planes). While the dispersions are similar to noninteracting ones, the hopping integrals $t, t^{\prime}$, and $t_{z}$ are renormalized by a factor of $\delta / 2$, where $\delta$ is a doping rate. The quantity $\Delta$ in Eq. (3) is a mean-field value of the bond field and is given by

$$
\Delta=\frac{J}{4 N_{s} N_{z}} \sum_{\mathbf{k}}\left(\cos k_{x}+\cos k_{y}\right) n_{F}\left(\varepsilon_{\mathbf{k}}\right),
$$

where $n_{F}\left(\varepsilon_{\mathbf{k}}\right)$ is the Fermi function, $N_{s}$ the total number of lattice sites on the square lattice, and $N_{z}$ the number of layers along the $z$ direction. For a given doping $\delta, \mu$ and $\Delta$ are determined self-consistently by solving Eq. (5) and

$$
(1-\delta)=\frac{2}{N_{s} N_{z}} \sum_{\mathbf{k}} n_{F}\left(\varepsilon_{\mathbf{k}}\right)
$$

Charge fluctuations included in the $t-J$ model Eq. (1) are described by the $6 \times 6$ matrix of the bosonic propagator at the order of $1 / N$ :

$$
\left[D_{a b}\left(\mathbf{q}, i v_{n}\right)\right]^{-1}=\left[D_{a b}^{(0)}\left(\mathbf{q}, i v_{n}\right)\right]^{-1}-\Pi_{a b}\left(\mathbf{q}, i v_{n}\right),
$$

where $a$ and $b$ run from 1 to 6 ; $\mathbf{q}$ is a three-dimensional wave vector and $v_{n}$ is a bosonic Matsubara frequency. $D_{a b}^{(0)}\left(\mathbf{q}, i v_{n}\right)$ 
is the bare bosonic propagator and is obtained as

$$
\left[D_{a b}^{(0)}\left(\mathbf{q}, i v_{n}\right)\right]^{-1}=N\left(\begin{array}{cccccc}
\frac{\delta^{2}}{2}[V(\mathbf{q})-J(\mathbf{q})] & \frac{\delta}{2} & 0 & 0 & 0 & 0 \\
\frac{\delta}{2} & 0 & 0 & 0 & 0 & 0 \\
0 & 0 & \frac{4}{J} \Delta^{2} & 0 & 0 & 0 \\
0 & 0 & 0 & \frac{4}{J} \Delta^{2} & 0 & 0 \\
0 & 0 & 0 & 0 & \frac{4}{J} \Delta^{2} & 0 \\
0 & 0 & 0 & 0 & 0 & \frac{4}{J} \Delta^{2} .
\end{array}\right)
$$

Here $J(\mathbf{q})=\frac{J}{2}\left(\cos q_{x}+\cos q_{y}\right)$ and $V(\mathbf{q})$ is the long-range Coulomb interaction in momentum space for a layered system,

$$
V(\mathbf{q})=\frac{V_{c}}{A\left(q_{x}, q_{y}\right)-\cos q_{z}},
$$

where $V_{c}=e^{2} d\left(2 \epsilon_{\perp} a^{2}\right)^{-1}$ and

$$
A\left(q_{x}, q_{y}\right)=\alpha\left(2-\cos q_{x}-\cos q_{y}\right)+1 .
$$

The Coulomb interaction $V(\mathbf{q})$ is easily obtained by solving Poisson's equation on the lattice [49]. In Eq. (10), $\alpha=\frac{\tilde{\epsilon}}{(a / d)^{2}}$ and $\tilde{\epsilon}=\epsilon_{\|} / \epsilon_{\perp} ; \epsilon_{\|}$and $\epsilon_{\perp}$ are the dielectric constants parallel and perpendicular to the planes, respectively; $e$ is the electric charge of electrons.

The $6 \times 6$ matrix $\Pi_{a b}$ is the bosonic self-energy

$$
\Pi_{a b}\left(\mathbf{q}, i v_{n}\right)=-\frac{N}{N_{s} N_{z}} \sum_{\mathbf{k}} h_{a}\left(\mathbf{k}, \mathbf{q}, \varepsilon_{\mathbf{k}}-\varepsilon_{\mathbf{k}-\mathbf{q}}\right) \frac{n_{F}\left(\varepsilon_{\mathbf{k}-\mathbf{q}}\right)-n_{F}\left(\varepsilon_{\mathbf{k}}\right)}{i v_{n}-\varepsilon_{\mathbf{k}}+\varepsilon_{\mathbf{k}-\mathbf{q}}} h_{b}\left(\mathbf{k}, \mathbf{q}, \varepsilon_{\mathbf{k}}-\varepsilon_{\mathbf{k}-\mathbf{q}}\right)-\delta_{a 1} \delta_{b 1} \frac{N}{N_{s} N_{z}} \sum_{\mathbf{k}} \frac{\varepsilon_{\mathbf{k}}-\varepsilon_{\mathbf{k}-\mathbf{q}}}{2} n_{F}\left(\varepsilon_{\mathbf{k}}\right),
$$

where the six-component vertex is given by

$$
\begin{aligned}
h_{a}(\mathbf{k}, \mathbf{q}, v)= & \left\{\frac{2 \varepsilon_{\mathbf{k}-\mathbf{q}}+v+2 \mu}{2}+2 \Delta\left[\cos \left(k_{x}-\frac{q_{x}}{2}\right) \cos \left(\frac{q_{x}}{2}\right)+\cos \left(k_{y}-\frac{q_{y}}{2}\right) \cos \left(\frac{q_{y}}{2}\right)\right]\right. \\
& \left.1 ;-2 \Delta \cos \left(k_{x}-\frac{q_{x}}{2}\right) ;-2 \Delta \cos \left(k_{y}-\frac{q_{y}}{2}\right) ; 2 \Delta \sin \left(k_{x}-\frac{q_{x}}{2}\right) ; 2 \Delta \sin \left(k_{y}-\frac{q_{y}}{2}\right)\right\} .
\end{aligned}
$$

Here the dependence on $k_{z}$ and $q_{z}$ enters only through $\epsilon_{\mathbf{k}-\mathbf{q}}$ in the first column and the other columns contain the in-plane momentum only.

Charge fluctuation spectra are obtained by the analytical continuation in Eq. (7),

$$
i v_{n} \rightarrow v+i \Gamma_{\mathrm{ch}},
$$

where $\Gamma_{\mathrm{ch}}(>0)$ is infinitesimally small. By studying $\operatorname{Im} D_{a b}(\mathbf{q}, v)$, we can elucidate all possible charge dynamics in the layered $t-J$ model as was already performed in the literature [13].

If we set $J=0$, we would obtain $\Delta=0$ and all fluctuations associated with the bond field vanish. The bosonic propagator $D_{a b}$ is reduced to a $2 \times 2$ matrix with $a, b=$ 1,2 and only the usual on-site charge fluctuations are active [see also Eq. (A15)]. In fact, the element $(1,1)$ of $D_{a b}$ is related to the usual charge-charge correlation function [44]. $D_{22}$ and $D_{12}$ correspond to fluctuations associated with the non-double-occupancy condition and correlations between the non-double-occupancy condition and charge density fluctuations, respectively. When $J$ is finite, bond-charge fluctuations become active and $a$ and $b$ take values from 1 to 6 . Thus, both on-site charge and bond-charge fluctuations are present for a realistic situation.

Although each lattice site in the $t-J$ model corresponds to a $\mathrm{Cu}$ atom in the $\mathrm{CuO}_{2}$ plane, the effect of $\mathrm{O}$ atoms is implicitly included because the $t$ - $J$ model is derived from the three-band Hubbard model in the strong coupling limit [50]. Thus, naively speaking, the on-site charge fluctuations in the $t-J$ model, which is described by the $2 \times 2$ sector of $D_{a b}$, correspond to charge fluctuations on each $\mathrm{Cu}$ site, whereas the bond-charge fluctuations described by $D_{a b}$ with $a, b=3-6$ account for charge fluctuations between $\mathrm{Cu}$ sites, i.e., at the $\mathrm{O}$ sites. However, this does not mean that the on-site charge and bond-charge fluctuations can be considered only around $\mathrm{Cu}$ sites and $\mathrm{O}$ sites, respectively, because $\mathrm{Cu}$ orbitals strongly hybridize with $\mathrm{O}$ orbitals, forming the Zhang-Rice singlet [50].

Next, we compute the electron self-energy. At the order of $1 / N$, the imaginary part of the self-energy is obtained in a compact form as [48]

$$
\begin{aligned}
\operatorname{Im} \Sigma(\mathbf{k}, \omega)= & \frac{-1}{N_{s} N_{z}} \sum_{\mathbf{q}} \sum_{a, b} \operatorname{Im} D_{a b}(\mathbf{q}, v) h_{a}(\mathbf{k}, \mathbf{q}, v) h_{b}(\mathbf{k}, \mathbf{q}, v) \\
& \times\left[n_{F}\left(-\varepsilon_{\mathbf{k}-\mathbf{q}}\right)+n_{B}(v)\right],
\end{aligned}
$$

where $v=\omega-\varepsilon_{\mathbf{k}-\mathbf{q}}$ and $n_{B}(v)$ is the Bose function. Note that the self-energy effects arise from charge fluctuations described by $D_{a b}$ and the effect of spin fluctuations does not appear at $O(1 / N)$. The form of $\operatorname{Im} \Sigma(\mathbf{k}, \omega)$ in Eq. (14) has the same structure as a self-energy obtained from the Fock diagram in a usual perturbation theory. However, in the 
large- $N$ scheme, we have both Hartree and Fock diagrams in a nontrivial way at order of $1 / N$; see Appendix A for further details.

Using the Kramers-Kronig relations, we obtain $\operatorname{Re} \Sigma(\mathbf{k}, \omega)$ from $\operatorname{Im} \Sigma(\mathbf{k}, \omega)$. Since the electron Green's function $G(\mathbf{k}, \omega)$ is given by

$$
G^{-1}(\mathbf{k}, \omega)=\omega+i \Gamma_{\mathrm{sf}}-\varepsilon_{\mathbf{k}}-\Sigma(\mathbf{k}, \omega),
$$

we can compute the one-particle spectral function $A(\mathbf{k}, \omega)=$ $-\frac{1}{\pi} \operatorname{Im} G(\mathbf{k}, \omega)$ as

$$
\begin{aligned}
& A(\mathbf{k}, \omega) \\
& \quad=-\frac{1}{\pi} \frac{\operatorname{Im} \Sigma(\mathbf{k}, \omega)-\Gamma_{\mathrm{sf}}}{\left[\omega-\varepsilon_{\mathbf{k}}-\operatorname{Re} \Sigma(\mathbf{k}, \omega)\right]^{2}+\left[-\operatorname{Im} \Sigma(\mathbf{k}, \omega)+\Gamma_{\mathrm{sf}}\right]^{2}},
\end{aligned}
$$

where $\Gamma_{\mathrm{sf}}(>0)$ originates from the analytical continuation in the electron Green's function.

The real part $\operatorname{Re} \Sigma(\mathbf{k}, \omega)$ contains a frequency-independent contribution and shifts the band dispersion. Since the dispersion in Eq. (2) is chosen to reproduce the experimental Fermi surface, we drop this frequency-independent contribution to avoid double counting. This procedure does not affect anything on $\operatorname{Im} \Sigma(\mathbf{k}, \omega)$, which can be easily understood from the Kramers-Kronig relations.

We have formulated a theory in the large- $N$ scheme to compute the electron self-energy at the order of $1 / N$. This kind of calculation is not seen much in literature. For example, the large- $N$ theory is often formulated in the slave-boson method. However there are few calculations including fluctuations above the mean field [46,51], and many calculations were performed at the mean-field level [51-53].

\section{RESULTS}

Following our previous works, which capture the charge dynamics in e-cuprates in both high- [13,16-18,29] and lowenergy regions [13-15] consistently, we choose the band parameters as $t^{\prime} / t=0.3, t_{z} / t=0.1$. The other parameters are taken to be $J / t=0.3, V_{c} / t=17, \alpha=4.5$, and $N_{z}=10$. The resulting momentum along the $z$ direction is given by a discrete value such as $q_{z}=2 \pi n_{z} / 10$ with $n_{z}$ being integer. While a value of $\Gamma_{\text {ch }}$ is infinitesimally small in Eq. (13), we set $\Gamma_{\mathrm{ch}} / t=0.03$ for numerical reasons. This finite $\Gamma_{\mathrm{ch}}$ may mimic phenomenologically a broadening of the spectrum [17] due to the resolution of experimental measurements and also additional effects not included in the present theory such as higher-order electron correlation effects [54] and coupling to other degrees of freedom. Temperature is set at $T=0$ and we focus on quantum charge fluctuations. We first present results for the doping rate $\delta=0.15$ and then study the doping dependence later. When computing the spectral function, we choose $\Gamma_{\text {sf }} / t=0.01$ to make the spectrum reasonably broadened.

Complete information of the charge dynamics is included in the $6 \times 6$ matrix $D_{a b}$ in Eq. (7), where both on-site charge and bond-charge fluctuations are present. As we shall show below, the major contributions to the electron self-energy come from the on-site charge fluctuations described by the $2 \times 2$ sector of $D_{a b}$, namely, $a, b=1,2$ in Eq. (14). Hence, we first focus on results from on-site charge fluctuations and
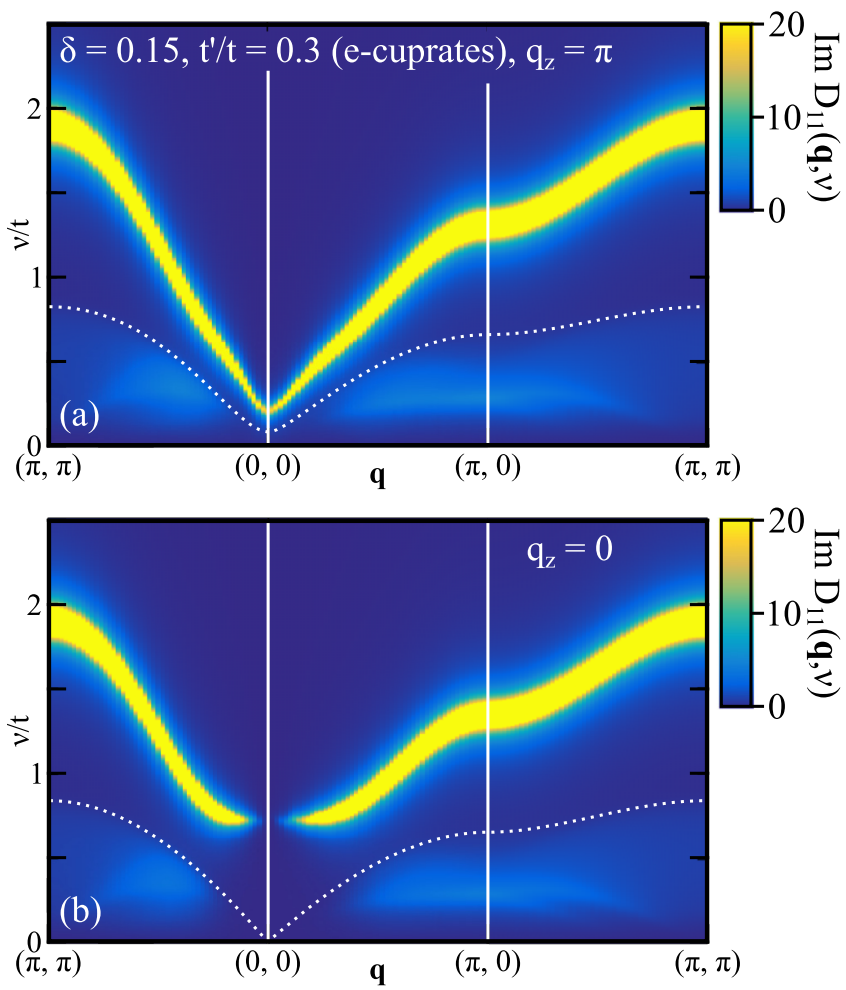

FIG. 1. Typical charge excitation spectrum [intensity map of $\left.\operatorname{Im} D_{11}(\mathbf{q}, v)\right]$ in the plane of momentum $\mathbf{q}$ and energy $v$ for $q_{z}=\pi$ (a) and $q_{z}=0$ (b). The result for $q_{z}=\pi$ is a representative one of results for other $q_{z}(\neq 0)$. The dotted curve is the upper limit of the continuum excitations.

clarify the major features of the electron self-energy due to the coupling to them. The effect of bond-charge fluctuations and a possible pseudogap feature are studied afterward. We also study a different set of band parameters appropriate to h-cuprates. We mention some care about our results for ecuprates when we compare them with experiments. Finally, we present results in the absence of the long-range Coulomb interaction to highlight its role in the electron self-energy.

\section{A. Charge excitation spectrum}

The charge excitation spectra $\operatorname{Im} D_{a b}(\mathbf{q}, v)$ are already presented for all components $a$ and $b$ in Figs. 1 and 8 in Ref. [13]. Furthermore, detailed analyses were performed for both the on-site charge excitations [13,16-18] and the bond-charge excitations [13-15]. For the sake of readers' convenience, we here summarize the typical features of charge excitation spectra relevant to the understanding of the electron self-energy.

The diagonal components of $\operatorname{Im} D_{a b}(\mathbf{q}, v)$ are always positive, as they should be. Figure 1 shows a typical charge-excitation spectrum by choosing $a=b=1$. The sharp features correspond to plasmon excitations. In particular, they exhibit a V-shaped dispersion around $\mathbf{q}=\left(0,0, q_{z}\right)$ with $q_{z} \neq 0$ [Fig. 1(a)]. The gap at $\mathbf{q}=\left(0,0, q_{z}\right)$ is proportional to the interlayer hopping integral $t_{z}$ (Refs. [16,41,43]). For $q_{z}=0$ [Fig. 1(b)], instead, we obtain the well-known optical plasmon with a nearly flat dispersion around $\omega=0$. In the 


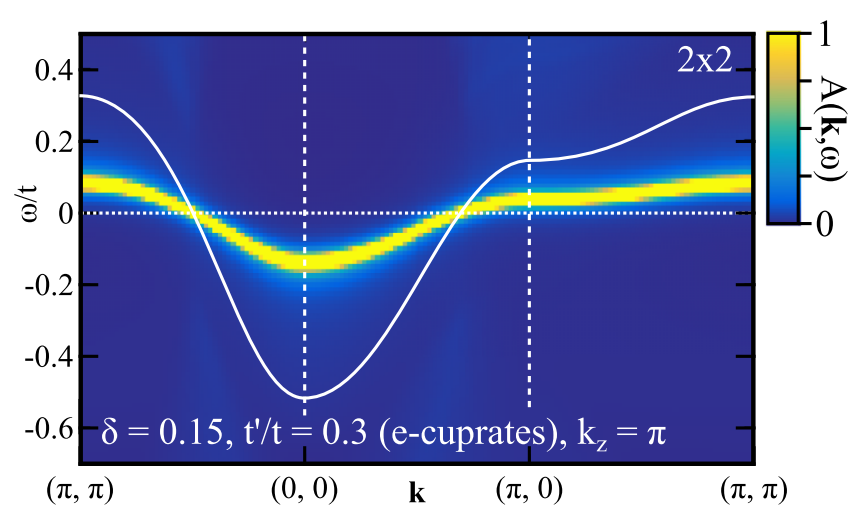

FIG. 2. Intensity map of $A(\mathbf{k}, \omega)$ along the direction $(\pi, \pi)$ $(0,0)-(\pi, 0)-(\pi, \pi)$ for $k_{z}=\pi$. The quasiparticle dispersion in the presence of the self-energy is in yellow. The white curve is the quasiparticle dispersion obtained in leading order theory without the self-energy [Eq. (2)]. See also Fig. 12 (a) obtained after a particlehole transformation.

low-energy region, namely, below the dotted line in Fig. 1, the so-called particle-hole continuum is realized. No strong spectral weight appears inside the continuum and there is no tendency toward conventional charge-density-wave order including stripes. When we choose $a=b=3-6$ and explore bond-charge excitations, we find strong spectral weight in the low-energy region inside the continuum [13]. However, the effect of the bond-charge excitations on the electron self-energy is minor as we shall show below.

The off-diagonal components $\operatorname{Im} D_{a b}(\mathbf{q}, v)$ are not positive definite any longer (see Figs. 1 and 8 in Ref. [13]). Typically they exhibit strong spectral weight in almost the same $(\mathbf{q}, v)$ region where $\operatorname{Im} D_{11}(\mathbf{q}, v)$ exhibit the plasmon dispersion.

\section{B. Self-energy effect from on-site charge fluctuations}

The quasiparticle dispersion in leading order theory [Eq. (2)] is plotted with a white curve in Fig. 2, where the hopping integrals are already renormalized and the energy scale is reduced to $J(=0.3 t)$. The quasiparticle dispersion is further renormalized by the coupling to the on-site charge fluctuations. To see that, we show in Fig. 2 the intensity map of $A(\mathbf{k}, \omega)$ [Eq. (16)] around the Fermi energy for $k_{z}=\pi$; the $k_{z}$ dependence is weak and essentially the same results are obtained for a different value of $k_{z}$. The bandwidth is suppressed significantly to become less than half, indicating that the quasiparticle weight $Z$ is reduced substantially by the on-site charge fluctuations.

Figure 3 shows the imaginary part of the electron selfenergy $\operatorname{Im} \Sigma(\mathbf{k}, \omega)$ as a function of energy $\omega$ for several choices of momenta $\mathbf{k}$. The self-energy exhibits a pronounced asymmetry with respect to $\omega=0$ and is strongly suppressed in $\omega>0$, leading to substantial breaking of the particle-hole symmetry. This asymmetry does not come from the band structure effect due to the presence of $t^{\prime}$, but from the strong correlation effect due to the local constraint inherent in the $t-J$ model. In fact, calculations in the random phase approximation (RPA) predict that $\operatorname{Im} \Sigma(\mathbf{k}, \omega)$ is rather symmetric with respect to $\omega=0$ (Ref. [55]). Hence, the analysis in RPA would lead to insights different from the present paper.

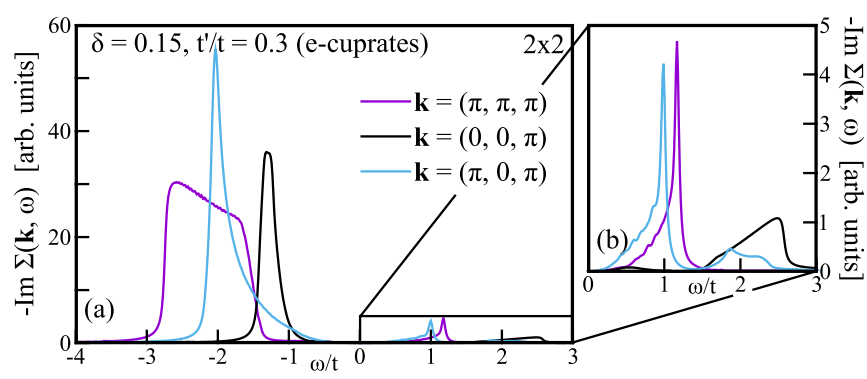

FIG. 3. Imaginary part of the electron self-energy, $-\operatorname{Im} \Sigma(\mathbf{k}, \omega)$, as a function of $\omega$ for several choices of $\mathbf{k}$. The positive energy region is magnified in the right panel. Results do not depend much on a value of $k_{z}$ and $k_{z}=\pi$ is taken as a representative one. See also Fig. 15 obtained after a particle-hole transformation.

To understand the reason for the particle-hole asymmetry in Fig. 3, we go back to Eq. (14). First, as we clarified in Ref. [13] and have also shown in Fig. 1, the charge excitation spectrum $\operatorname{Im} D_{a b}(\mathbf{q}, v)$ with $a, b=1,2$ has a sizable spectral weight in $v / t \lesssim 1$ around $\mathbf{q}=\left(0,0, q_{z}\right)$ and in a higher energy region typically around $\mathbf{q}=\left(\pi, \pi, q_{z}\right)$; the $q_{z}$ dependence is minor. $\operatorname{Im} D_{a b}(\mathbf{q}, v)$ is an odd function with respect to $v$. For $v>0$, we have $\operatorname{Im} D_{a a}(\mathbf{q}, v) \geqslant 0$ for the diagonal components and $\operatorname{Im} D_{a b}(\mathbf{q}, v) \leqslant 0$ for the off-diagonal components (see Figs. 1 and 8 in Ref. [13]). The vertex function is given by $h_{1}(\mathbf{k}, \mathbf{q}, v) \propto v$ for a large $v$ and $h_{2}(\mathbf{k}, \mathbf{q}, v)=1$; see Eq. (12). We consider three different values of $\mathbf{k}$ as typical ones: $\mathbf{k}=$ $\left(0,0, k_{z}\right),\left(\pi, \pi, k_{z}\right)$, and $\left(\pi, 0, k_{z}\right)$. The term of $n_{F}\left(-\varepsilon_{\mathbf{k}-\mathbf{q}}\right)+$ $n_{B}(v)$ takes a constant value depending on $\mathbf{k}, \mathbf{q}$, and $v$ as summarized in Table I, which works as a selection rule. Note that for $v<0$, the term $n_{F}\left(-\varepsilon_{\mathbf{k}-\mathbf{q}}\right)+n_{B}(v)$ can become negative, but the sign of $\operatorname{Im} D_{a b}(\mathbf{q}, v)$ also changes; consequently, their product does not change the sign. Considering all these features, we recognize that all components $a, b=1,2$ contribute additively to the summation for $v<0$ in Eq. (14) whereas the contribution from the diagonal components $a=b$ is largely canceled by the off-diagonal components $a \neq b$ for $v>0$, leading to the strong asymmetry of the self-energy as shown in Fig. 3. The off-diagonal components of $\operatorname{Im} D_{a b}(\mathbf{q}, v)$ originate from the strong correlation effect related to the Lagrange multiplier describing the nondouble occupancy of electrons at any site on the lattice.

Recalling that we have the relation $v=\omega-\varepsilon_{\mathbf{k}-\mathbf{q}}$ in Eq. (14) and the value of $\left|\varepsilon_{\mathbf{k}-\mathbf{q}}\right|$ is less than $1 t$ whereas the typical energy range that we are interested in Fig. 3 is more

TABLE I. Typical values of $n_{F}\left(-\varepsilon_{\mathbf{k}-\mathbf{q}}\right)+n_{B}(v)$ at $T=0$. They may depend sensitively on the precise values of $\mathbf{k}$ and $\mathbf{q}$ for $\mathbf{k} \sim\left(\pi, 0, k_{z}\right)$ because the Fermi surface is located rather close to $\mathbf{k}=\left(\pi, 0, k_{z}\right)$.

\begin{tabular}{|c|c|c|c|c|}
\hline \multirow[t]{2}{*}{$\mathbf{k}$} & \multicolumn{2}{|c|}{$\sim\left(0,0, q_{z}\right)$} & \multicolumn{2}{|c|}{$\sim\left(\pi, \pi, q_{z}\right)$} \\
\hline & $v<0$ & $v>0$ & $v<0$ & $v>0$ \\
\hline$\sim\left(0,0, k_{z}\right)$ & -1 & 0 & 0 & 1 \\
\hline$\sim\left(\pi, \pi, k_{z}\right)$ & 0 & 1 & -1 & 0 \\
\hline$\sim\left(\pi, 0, k_{z}\right)$ & -1 or 0 & 0 or 1 & -1 or 0 & 0 or 1 \\
\hline
\end{tabular}


than $1 t$, the sign of $v$ becomes the same as that of $\omega$ independent of $\mathbf{k}$ and $\mathbf{q}$ in most cases. Hence by associating the sign of $\omega$ with that of $v$, the selection rule in Table I serves to identify the origin of the structure of $\operatorname{Im} \Sigma(\mathbf{k}, \omega)$. For $\omega>0$, the peak around $\omega / t \approx 1$ for $\mathbf{k}=\left(\pi, \pi, k_{z}\right)$ comes from the plasmon excitations around $\mathbf{q} \sim\left(0,0, q_{z}\right)$. A long tail on a lower energy side of the peak originates from the acousticlike plasmon mode. The structure around $\omega / t \approx 2.5$ for $\mathbf{k}=\left(0,0, k_{z}\right)$ comes mainly from charge excitations around $\mathbf{q} \sim\left(\pi, \pi, q_{z}\right)$. For an intermediate momentum such as $\mathbf{k}=$ $\left(\pi, 0, q_{z}\right), \operatorname{Im} \Sigma(\mathbf{k}, \omega)$ exhibits typically two structures around $\omega / t \approx 1$ and 2 ; the former stems from plasmons and the latter from charge excitations around $\mathbf{q} \sim\left(\pi, \pi, q_{z}\right)$. For $\omega<0$, on the other hand, the major contribution of charge fluctuations becomes vice versa: charge fluctuations around $\mathbf{q} \sim\left(0,0, q_{z}\right)$ and $\left(\pi, \pi, q_{z}\right)$ form the structure around $\omega / t \approx-1$ for $\mathbf{k}=$ $\left(0,0, k_{z}\right)$ and $\omega / t \approx-2$ for $\mathbf{k}=\left(\pi, \pi, k_{z}\right)$, respectively. For an intermediate momentum $\mathbf{k}=\left(\pi, 0, k_{z}\right)$, essentially a single peak is realized around $\omega / t \approx-2$ with a sizable tail on the side of $\omega=0$.

In Fig. 4, we summarize $\operatorname{Im} \Sigma(\mathbf{k}, \omega), \operatorname{Re} \Sigma(\mathbf{k}, \omega)$, and $A(\mathbf{k}, \omega)$ as a function of $\omega$ for $\mathbf{k}=(0,0, \pi),(\pi, \pi, \pi)$, and $(\pi, 0, \pi)$. Since $A(\mathbf{k}, \omega)$ can form a peak when the following equation

$$
\omega-\varepsilon_{\mathbf{k}}-\operatorname{Re} \Sigma(\mathbf{k}, \omega)=0
$$

is fulfilled [see Eq. (16)], we also add a line of $\omega-\varepsilon(\mathbf{k})$ in Fig. 4. The line crosses the curve of $\operatorname{Re} \Sigma(\mathbf{k}, \omega)$ typically at three points for each $\mathbf{k}$. One is very close to $\omega=0$ and yields the quasiparticle dispersion as already shown in Fig. 2. The spectral function $A(\mathbf{k}, \omega)$ exhibits a sharp peak there. The second one is close to the energy where $\operatorname{Im} \Sigma(\mathbf{k}, \omega)$ has a peak, leading to a strong damping. As a result, $A(\mathbf{k}, \omega)$ yields a very broad structure. The third one appears deeply below the Fermi energy, i.e., around $\omega / t \approx-4,-7$, and -5 in Figs. 4(a)-4(c), respectively, where $\operatorname{Im} \Sigma(\mathbf{k}, \omega)$ almost vanishes and thus $A(\mathbf{k}, \omega)$ forms a very sharp peak.

In addition, there are several subpeak structures in $A(\mathbf{k}, \omega)$. In $\omega>0, \operatorname{Im} \Sigma(\mathbf{k}, \omega)$ can form a structure in $1 \lesssim \omega / t \lesssim 2.5$ as already explained in Fig. 3, which yields a small peak structure of $\operatorname{Re} \Sigma(\mathbf{k}, \omega)$ there. The resulting value of $\mid \omega-$ $\varepsilon(\mathbf{k})-\operatorname{Re} \Sigma(\mathbf{k}, \omega) \mid$ [see Eq. (17)] is suppressed, leading to the subpeak structure of $A(\mathbf{k}, \omega)$. In particular, the peak around $\omega / t \approx 1$ is pronounced in Figs. 4(b) and 4(c).

Figure 5 shows the intensity map of $A(\mathbf{k}, \omega)$ in the plane of $\mathbf{k}$ and $\omega$. The quasiparticle band is realized around $\omega=0$. While it might look nondispersive in the energy scale in Fig. 5, it does disperse in a scale of $J(=0.3 t)$ as shown in Fig. 2. A sharp dispersion in $-7 \lesssim \omega / t \lesssim-4$ is an emergent band arising from the coupling to the on-site charge fluctuations. In the positive energy region, one clear dispersion emerges around $\omega / t \approx 1$, especially along the $(\pi, 0)-(\pi, \pi)-(\pi / 2, \pi / 2)$ direction. The origin of this band lies in the acousticlike and optical plasmons around $\mathbf{q}=\left(0,0, q_{z}\right)$ and is pronounced around $\mathbf{k}=\left(\pi, 0, k_{z}\right)$ and $\left(\pi, \pi, k_{z}\right)$, as we have explained in the context of Table I. The clear separation of this emergent band around $\omega / t \approx 1$ from the main quasiparticle band around $\omega \approx 0$ is due to the relatively large energy scale of plasmons around $\mathbf{q}=\left(0,0, q_{z}\right)$. If we discard the plasmons by replacing the long-range Coulomb interaction with a short-range one,
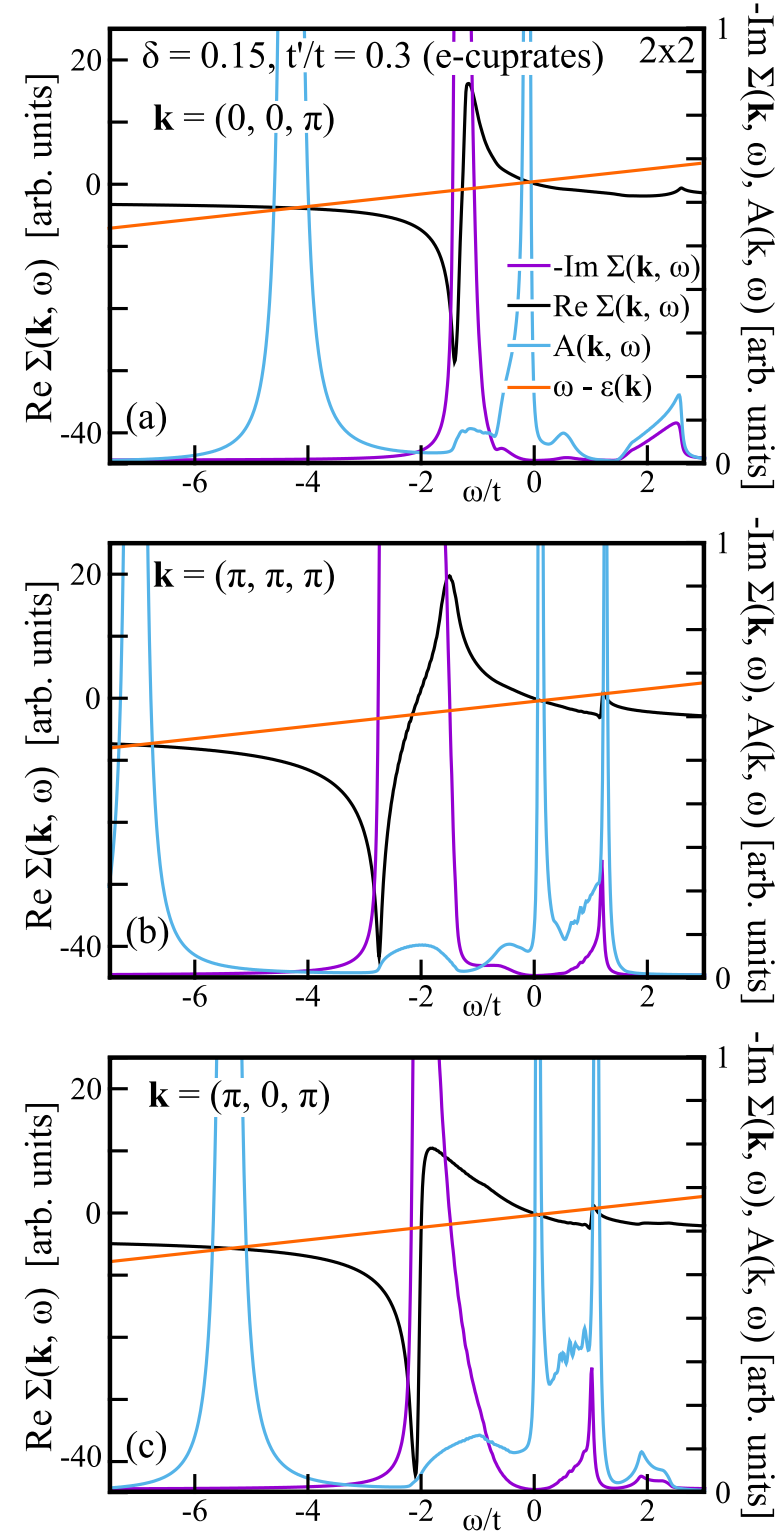

FIG. 4. Energy dependence of $-\operatorname{Im} \Sigma(\mathbf{k}, \omega), \operatorname{Re} \Sigma(\mathbf{k}, \omega)$, and $A(\mathbf{k}, \omega)$ for $\mathbf{k}=(0,0, \pi)(\mathrm{a}),(\pi, \pi, \pi)(\mathrm{b})$, and $(\pi, 0, \pi)(\mathrm{c})$. The line of $\omega-\varepsilon(\mathbf{k})$ is also drawn. The scales of $-\operatorname{Im} \Sigma(\mathbf{k}, \omega)$ and $A(\mathbf{k}, \omega)$ correspond to the right vertical axis and their units are taken differently to clarify their peak structure in the same panel. See also Fig. 16 obtained after a particle-hole transformation.

the charge excitations are characterized by a zero-sound mode around $\mathbf{q}=\left(0,0, q_{z}\right)$ (see Ref. [56]), which becomes gapless at $\mathbf{q}=(0,0,0)$. In this case, an emergent band corresponding to that around $\omega / t \approx 1$ tends to merge into the main quasiparticle band (see Fig. 13).

There are also weak and fine structures in Fig. 5. Two weak bandlike features emerge in $-1 \lesssim \omega / t \lesssim 0$ on both sides of $\mathbf{k}=\left(0,0, k_{z}\right)$. These weak structures are due to the peak structure of $\operatorname{Im} \Sigma(\mathbf{k}, \omega)$ as explained in Fig. 4, yielding incoherent bands there. This incoherency comes from the coupling to the plasmons. Around $\omega / t \approx 2$ and $\mathbf{k} \approx\left(0,0, k_{z}\right)$, we also have a weak structure, which is barely visible in Fig. 5 . 


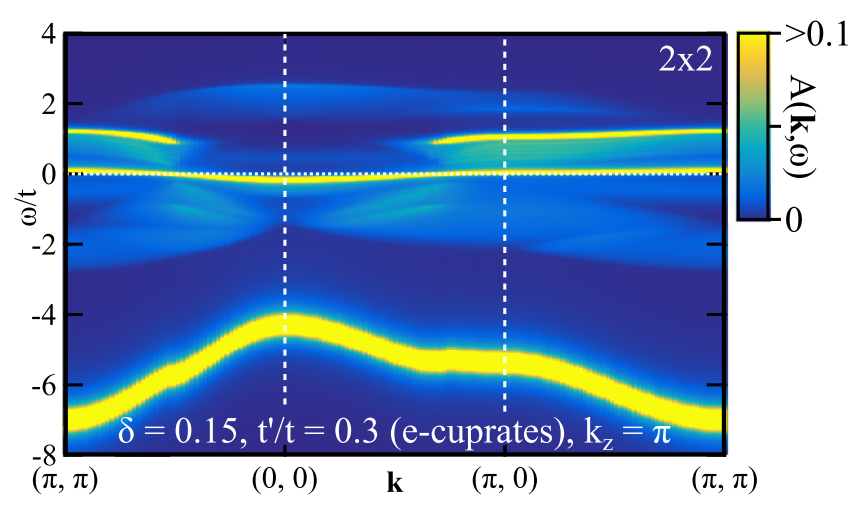

FIG. 5. Intensity map of $A(\mathbf{k}, \omega)$ along the direction $(\pi, \pi)$ $(0,0)-(\pi, 0)-(\pi, \pi) ; k_{z}$ dependence is weak and $k_{z}=\pi$ is taken as a representative value. See also Fig. 12(b) obtained after a particle-hole transformation.

This incoherent band originates from the coupling to charge excitations around $\mathbf{q} \sim\left(\pi, \pi, q_{z}\right)$.

In Fig. 6, we show how the density of states is renormalized by electron correlation effects in the $t-J$ model. Our original band Eq. (2) is already renormalized at leading order as seen in Fig. 2. Hence the density of states is limited to a narrow energy window in $-0.6 \lesssim \omega / t \lesssim 0.5$. The coupling to the on-site charge fluctuations $(2 \times 2$ in Fig. 6) then splits the density of states into three. The resulting energy window of the density of states around the Fermi energy $(\omega=0)$ shrinks substantially and two states are generated around $\omega / t \approx 1$ and -5.5 .

\section{Effect of bond-charge fluctuations and doping dependence}

So far, we have restricted the summation of $a, b$ in Eq. (14) to $a, b=1,2$, that is, we have focused on the effect of the on-site charge fluctuations, which yield plasmons [13,16-18,29]. As emphasized in Ref. [13], charge excitations in the $t-J$ model contain not only the on-site charge fluc-

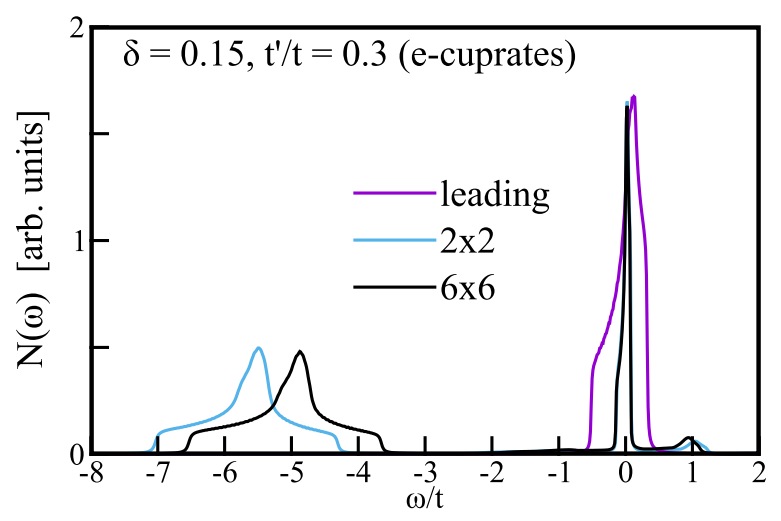

FIG. 6. The density of states for three difference cases: one is at leading order in the large- $N$ theory [Eq. (2)] and the other two are $2 \times 2$ and $6 \times 6$, where the self-energy effect is taken into account from on-site charge fluctuations and both on-site charge and bondcharge fluctuations, respectively. The results for $2 \times 2$ and $6 \times 6$ almost overlap with each other around $\omega / t=0$ and 1 . See also Fig. 12(d) obtained after a particle-hole transformation.

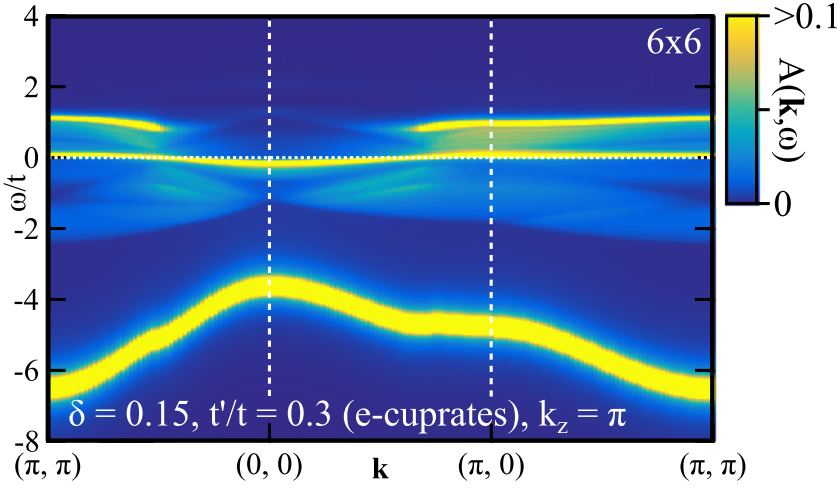

FIG. 7. Intensity map of $A(\mathbf{k}, \omega)$ along the direction $(\pi, \pi)$ $(0,0)-(\pi, 0)-(\pi, \pi)$ in the presence of both on-site charge and bond-charge fluctuations. $k_{z}$ dependence is weak and $k_{z}=\pi$ is taken as a representative value. See also Fig. 12(c) obtained after a particlehole transformation.

tuations but also bond-charge fluctuations. The bond-charge fluctuations are incorporated by allowing the indices $a$ and $b$ up to 6 in Eq. (14). The obtained spectral function $A(\mathbf{k}, \omega)$ is shown in Fig. 7. A comparison with Fig. 5 demonstrates that essentially the same results are obtained even if the bondcharge fluctuations are taken into account. A close inspection reveals a suppression of an incoherent band around $\omega / t \approx 2$ and $\mathbf{k} \approx\left(0,0, k_{z}\right)$ and the resulting band becomes invisible in Fig. 7. It can be said that the one-particle excitation spectrum $A(\mathbf{k}, \omega)$ is mainly controlled by the on-site charge fluctuations and the effect of the bond-charge fluctuations is minor.

This insight also applies to the density of states shown in Fig. 6. It is interesting that the bond-charge fluctuations impact the density of states in a high-energy region around $\omega / t \approx-5$ more than that in $-0.5 \lesssim \omega / t \lesssim 1$, although the typical energy scale of the bond-charge excitations is around $J$.

Bond-charge fluctuations become stronger when the system is tuned closer to a bond-charge instability. However, their effect on the electron self-energy is still minor compared with the on-site charge fluctuations. To demonstrate this, we study how the quasiparticle weight is renormalized via coupling to charge fluctuations. In the present theory with $\Gamma_{\mathrm{ch}} / t=0.03$ [Eq. (13)], $d$-wave bond-charge ( $d$ bond) order [57-63] and flux order $[53,64,65]$, the so-called $d$-wave charge density wave $(d \mathrm{CDW})$ [66], are stabilized below $\delta_{c} \approx 0.06$ and 0.07 , respectively, at $T=0$. The value of $\delta_{c}$ depends strongly on a choice of $\Gamma_{\mathrm{ch}}$, and Refs. [19,45] studied charge instabilities for $\Gamma_{\mathrm{ch}} / t=0.01$. Since the quasiparticle weight can depend on the Fermi momentum, we first show its typical dependence [Fig. 8(a)] by setting $\delta=0.15$, which is away from the bondcharge instabilities, and then clarify its doping dependence afterward [Fig. 8(b)].

In Fig. 8(a), the momentum dependence of $Z(\varphi)$ is presented along the Fermi surface; $\varphi$ is defined in the inset. $Z(\varphi)$ associated with $d \mathrm{CDW}$ is close to unity at $\varphi=\pi / 4$ and is reduced to around 0.7 upon approaching $\varphi=0$, showing the anisotropy of $Z(\varphi)$ with a $d$-wave symmetry. $Z(\varphi)$ associated with $d$ bond becomes smaller than the case of $d \mathrm{CDW}$ and is almost isotropic. This dependence might seem peculiar 

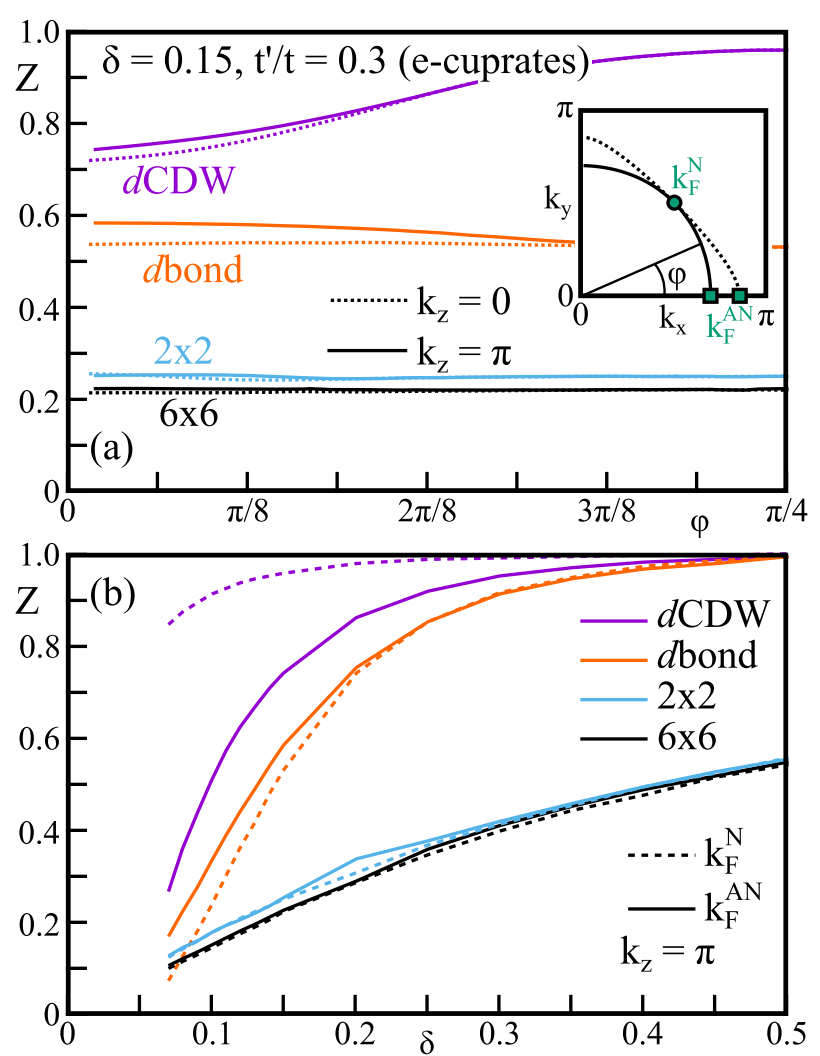

FIG. 8. (a) Quasiparticle weight $Z(\varphi)$ along the Fermi surface for $k_{z}=0$ and $\pi$ at $\delta=0.15$. The momentum is measured by the angle $\varphi$ defined in the inset. Four different cases are plotted: (i) $6 \times 6$, where full charge fluctuations are included, (ii) $2 \times 2$, where only on-site charge fluctuations are considered, (iii) $d$ bond and (iv) $d \mathrm{CDW}$, where fluctuations associated with $d$ bond and $d \mathrm{CDW}$ are considered, respectively. In the inset, Fermi surfaces shown by solid and dotted lines correspond to $k_{z}=\pi$ and 0 , respectively. (b) Doping dependence of $Z(\varphi)$ at $\varphi=0$ and $\pi / 4$ for four different cases. The $d \mathrm{CDW}$ and $d$ bond are stabilized below $\delta_{c} \approx 0.07$ and 0.06 , respectively. After the particle-hole transformation discussed in Sec. III F, the results stay intact except that the momentum in the inset in (a) should be transformed as $(0,0) \rightarrow(\pi, \pi)$ and $(\pi, \pi) \rightarrow(0,0)$.

because it is not characterized by $d$-wave symmetry although $d$ bond contains $d$-wave in its terminology. This puzzle is easily resolved by noting that $d$ bond fluctuations have low-energy spectral weight around the in-plane momentum $(0.8 \pi, 0.8 \pi)$; see Fig. 4(a) in Ref. [13]. In this case, the $d$-wave form factor enhances the contribution from the nodal region around $\mathbf{k}=\left(\pi / 2, \pi / 2, k_{z}\right)$ more than the antinodal region around $\mathbf{k}=\left(\pi, 0, k_{z}\right)$ and $\left(0, \pi, k_{z}\right)$. But it depends on the choice of parameters whether $Z$ at $\varphi=\pi / 4$ eventually becomes larger than $Z$ at $\varphi=0$.

On the other hand, $Z(\varphi)$ from the on-site charge fluctuations $[2 \times 2$ in Fig. $8($ a) $]$ is isotropic along the Fermi surface and becomes around 0.25 , much smaller than $Z$ from $d$ CDW and $d$ bond. In addition, $Z(\varphi)$ from $2 \times 2$ almost reproduces $Z(\varphi)$ from all charge fluctuations denoted by $6 \times 6$ in Fig. 8(a). This clearly indicates that the momentum dependence of $Z(\varphi)$ from $d$ bond and $d \mathrm{CDW}$ is fully smeared out by the isotropic contribution from the on-site charge fluctuations.
We also plot $Z(\varphi)$ for different values of $k_{z}$ in Fig. 8(a). $k_{z}$ dependence is visible for $d \mathrm{CDW}$ and $d$ bond, but is rather weak. On the other hand, it is negligible for $2 \times 2$ and $6 \times 6$.

The doping dependence of $Z(\varphi)$ is shown in Fig. 8(b) at two positions $\varphi=0$ and $\pi / 4$ for different charge fluctuations. With decreasing doping rate, $Z(\varphi)$ at $\varphi=0$ for $d \mathrm{CDW}$ is substantially suppressed whereas $Z(\varphi)$ at $\varphi=\pi / 4$ stays at a value near unity. The anisotropy of $Z(\varphi)$ is pronounced upon approaching the $d \mathrm{CDW}$ instability. The value of $Z(\varphi)$ for $d$ bond decreases at both $\varphi=0$ and $\pi / 4$ almost similarly with decreasing $\delta$ and their values become smaller than those for $d \mathrm{CDW}$.

For the purely on-site charge fluctuations denoted by $2 \times 2$ in Fig. 8(b), $Z(\varphi)$ is essentially isotropic in a whole doping region. Note that $Z(\varphi)$ decreases monotonically with decreasing $\delta$, although the number of carriers is decreased. The values of $Z(\varphi)$ for the full charge fluctuations denoted by $6 \times 6$ are almost the same as those for on-site charge fluctuations. This clearly indicates that the effect of the on-site charge fluctuations on the electron self-energy is dominant over the bond-charge fluctuations such as $d$ bond and $d$ CDW in a whole doping region.

\section{No pseudogap feature}

Figures 2 and 6 imply that charge fluctuations do not lead to a pseudogap feature. To confirm this implication, we show in Fig. 9(a) the spectral function at the nodal and antinodal Fermi momenta by focusing on the on-site charge fluctuations. Two pronounced peaks are realized: one is the quasiparticle peak at $\omega=0$ and the other corresponds to the emergent incoherent band near $\omega \approx 1 t$. This incoherent band is due to the coupling to the plasmons, as already explained in Sec. III B. There is a weak structure in $-1 \lesssim \omega / t \lesssim 0$, which is also recognized in the corresponding energy in Fig. 5. There is little difference between the nodal and antinodal Fermi momenta, demonstrating the isotropic renormalization effect from the on-site charge fluctuations.

The spectral function shown in Fig. 9(b) is obtained by taking full charge fluctuations into account, namely, both onsite charge and bond-charge fluctuations. A comparison with Fig. 9(a) shows that even though the typical energy scale of the bond-charge fluctuations is $J$, much lower than the on-site charge fluctuations, the effect of the bond-charge fluctuations are minor and no appreciable changes occur even around the Fermi energy.

Figure 9(c) is the intensity map of $A(\mathbf{k}, 0)$ at the Fermi energy in the first quadrant of the Brillouin zone. There is strong spectral weight entirely along the Fermi surface. A close look at Fig. 9(c) reveals that the spectrum becomes sharpest at the nodal direction and broadest at the antinodal region. This is due to the band structure effect, i.e., the proximity to the saddle points at $(\pi, 0)$ and $(0, \pi)$ in the electron dispersion [Eq. (3)]. Figures 2 and 6 together with Fig. 9 confirm no indication of a pseudogap feature in the presence of the quantum charge fluctuations in the $t-J$ model.

We checked that the essential features in Fig. 9 do not depend on doping (for $\delta>\delta_{c}$ ). Hence our Fermi surface is always large even though the system gets closer to a 

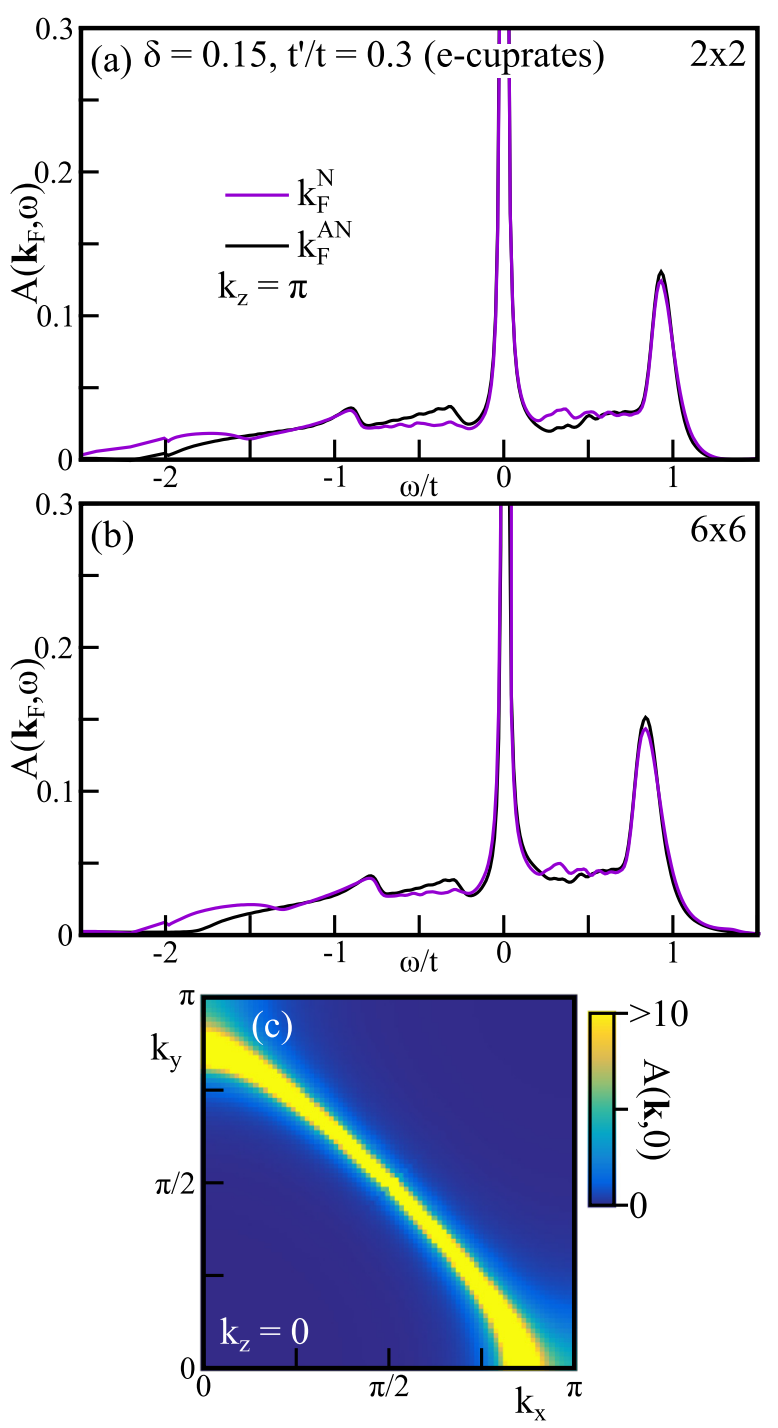

FIG. 9. (a), (b) Spectral function $A\left(\mathbf{k}_{F}, \omega\right)$ as a function of $\omega$ at the Fermi momentum; $k_{z}=\pi$ is taken as a representative one. $\mathbf{k}_{F}^{\mathrm{N}}$ and $\mathbf{k}_{F}^{\mathrm{AN}}$ are the Fermi momenta at the nodal $(\varphi=\pi / 4)$ and antinodal $(\varphi=0)$ points, respectively. In (a), only on-site charge fluctuations (denoted by $2 \times 2$ ) are considered whereas the effect of both on-site charge and bond-charge fluctuations $(6 \times 6)$ are taken into account in (b). (c) Intensity map of $A(\mathbf{k}, 0)$ at the Fermi energy in the first quadrant of the Brillouin zone for $k_{z}=0$ as a representative one. See also Figs. 12(e) and 12(f) obtained after a particle-hole transformation.

bond-charge instability at a lower doping and the spectral weight is suppressed substantially (Fig. 8).

\section{E. Results for h-cuprates}

So far we have presented results which can be applicable to e-cuprates. For parameters appropriate for h-cuprates, we obtain very similar results. Nonetheless, we think it worthwhile presenting the corresponding results for h-cuprates and clarifying the meaning of very similar results because h-cuprates are studied more than e-cuprates as a general trend. Following Ref. [17], we choose $t^{\prime} / t=-0.2$ and the same values as those
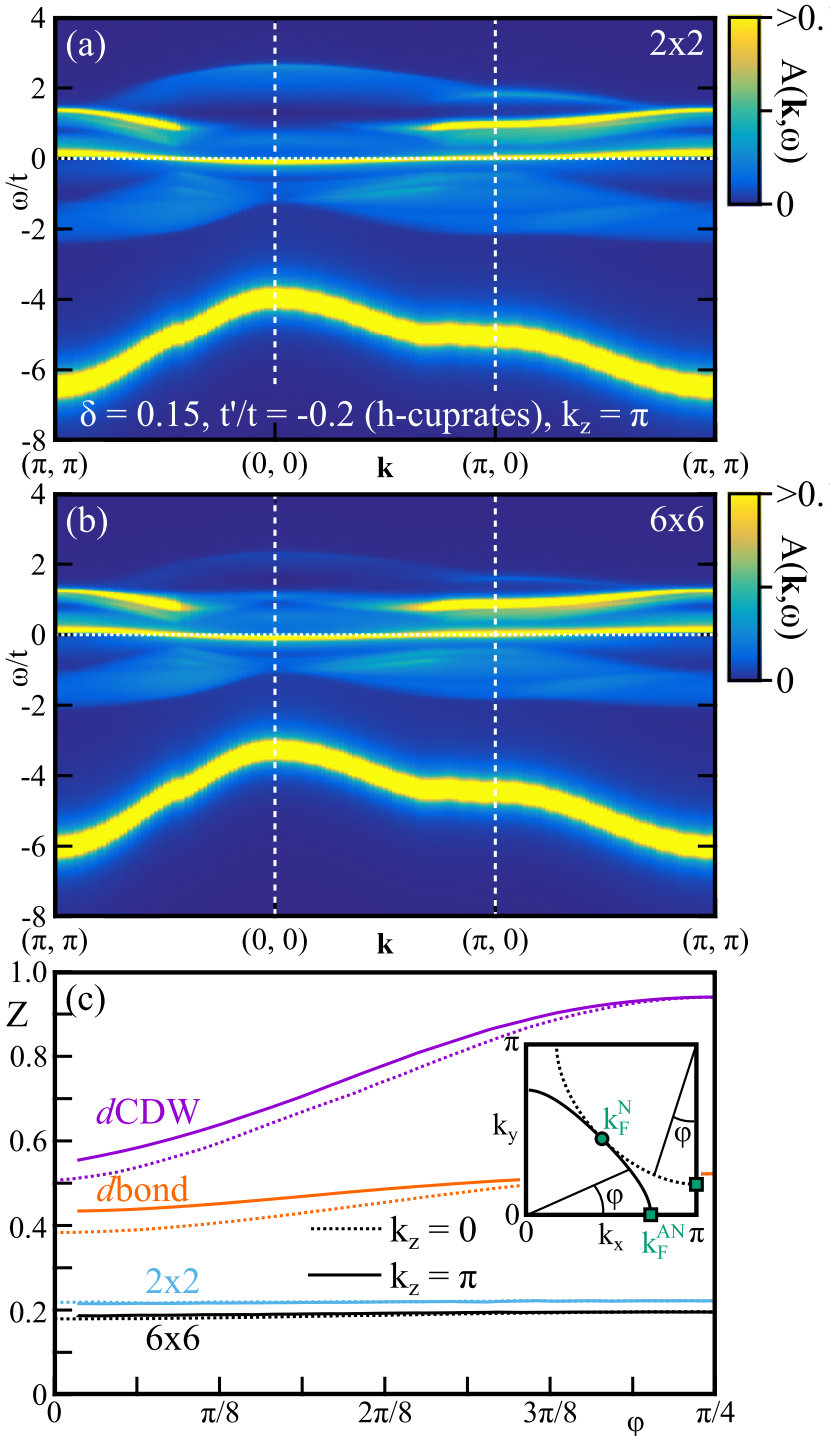

FIG. 10. Results for parameters appropriate for h-cuprates at $\delta=0.15$. (a) Intensity map of $A(\mathbf{k}, \omega)$ in the presence of the on-site charge fluctuations along the direction $(\pi, \pi)-(0,0)-(\pi, 0)-(\pi, \pi)$; $k_{z}$ dependence is weak and $k_{z}=\pi$ is taken as a representative value. (b) Intensity map of $A(\mathbf{k}, \omega)$ in the presence of both on-site charge and bond-charge fluctuations. (c) Quasiparticle weight $Z(\varphi)$ along the Fermi surface for four cases: $6 \times 6$ where full charge fluctuations are included, $2 \times 2$ where only on-site charge fluctuations are considered, and $d$ bond and $d \mathrm{CDW}$ where fluctuations associated with $d$ bond and $d \mathrm{CDW}$ are considered, respectively. The results for e-cuprates corresponding to (a)-(c) are shown in Figs. 5, 7, and 8(a), respectively.

for e-cuprates for the rest of the parameters such as $t_{z}, V_{c}, \alpha$, $J$, and $\delta(=0.15)$.

Figure $10(\mathrm{a})$ is the intensity map of $A(\mathbf{k}, \omega)$ due to the coupling to the on-site charge fluctuations and is practically the same as Fig. 5. This result does not change much even if we include the bond-charge fluctuations as shown in Fig. 10(b) and is very similar to Fig. 7. The electron self-energy is dominated by the on-site charge fluctuations, the same conclusion as that for e-cuprates. 


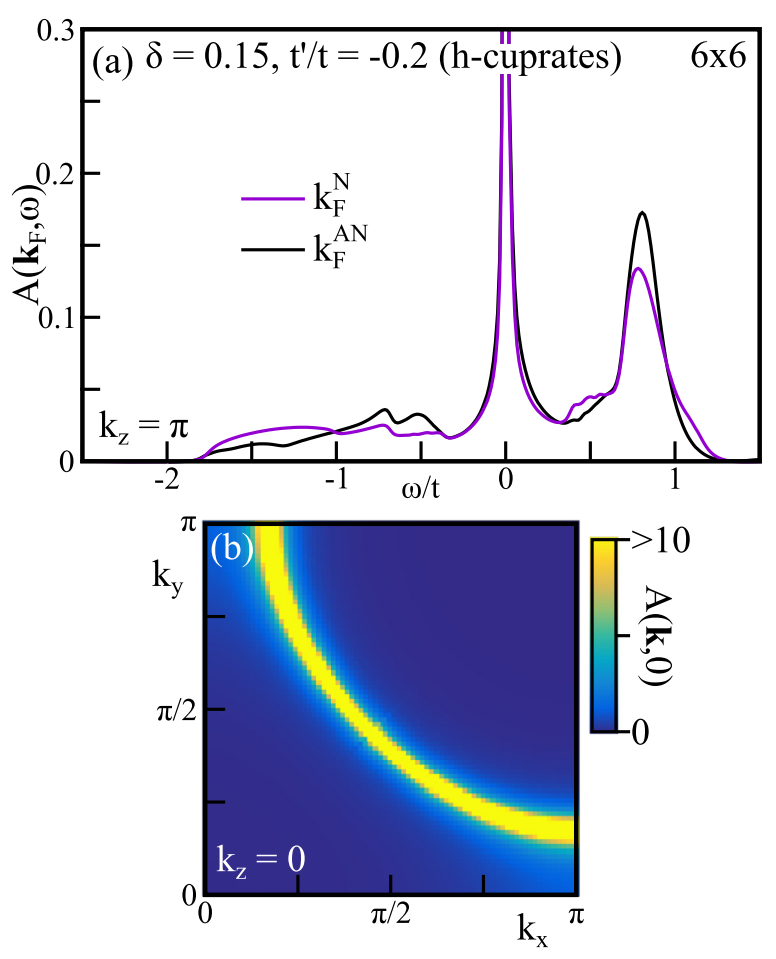

FIG. 11. Results for h-cuprates at $\delta=0.15$. (a) Spectral function $A\left(\mathbf{k}_{F}, \omega\right)$ as a function of $\omega$ at the Fermi momentum. $\mathbf{k}_{F}^{\mathrm{N}}$ and $\mathbf{k}_{F}^{\mathrm{AN}}$ are the Fermi momenta at the nodal $(\varphi=\pi / 4)$ and antinodal $(\varphi=0)$ points. (b) Intensity map of $A(\mathbf{k}, 0)$ at the Fermi energy in the first quadrant of the Brillouin zone. Corresponding results to e-cuprates are given in Figs. 9(b) and 9(c).

Figure 10(c) shows the quasiparticle weight $Z(\varphi)$ along the Fermi surface. This result is similar to the corresponding result for e-cuprates shown in Fig. 8(a). That is, the result for $2 \times 2$ is almost the same as that for $6 \times 6$, indicating that the effect of on-site charge fluctuations is dominant and $Z(\varphi)$ becomes almost isotropic along the Fermi surface. Since the present doping rate $\delta=0.15$ is close to the critical doping rate of the $d \mathrm{CDW}$ instability $\left(\delta_{c} \approx 0.12\right)$, the value of $Z(\varphi)$ is reduced more than that in Fig. 8(a) upon approaching $\varphi=0$. While the critical doping rate of the $d$ bond instability is almost the same as that for e-cuprates, the value of $Z(\varphi)$ away from $\varphi=\pi / 4$ and consequently its $\varphi$ dependence for $d$ bond become somewhat different from that in Fig. 8(a). This reflects the difference of the band structure. In fact, the $\varphi$ dependence of $Z$ for $d$ bond is rather sensitive to band parameters and a value of $Z$ at $\varphi=0$ can become larger or smaller than that at $\varphi=\pi / 4$. For the present parameters for h-cuprates, the inner Fermi surface becomes electronlike for $k_{z}=\pi$; see the inset in Fig. 10(c). It is remarkable that the difference of the Fermi surface topology between $k_{z}=0$ and $\pi$ does not produce appreciable changes of $Z(\varphi)$ not only for on-site charge fluctuations but also for bond-charge fluctuations in Fig. 10(c).

Figure 11(a) is the spectral function $A\left(\mathbf{k}_{F}, \omega\right)$ as a function of $\omega$ at the nodal $(\varphi=\pi / 4)$ and the antinodal $(\varphi=0)$ Fermi momenta, and Fig. 11(b) is the intensity map of $A(\mathbf{k}, 0)$ at the Fermi energy. These results should be compared with Figs. 9(b) and 9(c). No appreciable differences are recognized.
We can conclude that the quantum charge fluctuations do not lead to a pseudogap feature, even for h-cuprates.

\section{F. Particle-hole transformation of results for e-cuprates}

There is a special aspect of the $t-J$ model in a study of the one-particle properties for e-cuprates. That is, the e-cuprates are analyzed in terms of the hole picture in the $t-J$ model. This is because the $t-J$ model is defined in the restricted Hilbert space where the double occupancy of electrons is forbidden. Given that experimental data in e-cuprates are analyzed in the particle picture, it should be more transparent to present our results in the same picture.

We perform a particle-hole transformation in momentum space: $\tilde{c}_{\mathbf{k} \sigma} \rightarrow \tilde{c}_{\mathbf{k}+\mathbf{Q} \sigma}^{\dagger}$ and $\tilde{c}_{\mathbf{k} \sigma}^{\dagger} \rightarrow \tilde{c}_{\mathbf{k}+\mathbf{Q} \sigma}$ with $\mathbf{Q}=(\pi, \pi, 0)$ (Ref. [67]). This yields the following changes:

$$
\begin{aligned}
\epsilon_{\mathbf{k}} & \rightarrow-\epsilon_{\mathbf{k}+\mathbf{Q}}, \\
\operatorname{Re} \Sigma(\mathbf{k}, \omega) & \rightarrow-\operatorname{Re} \Sigma(\mathbf{k}+\mathbf{Q},-\omega), \\
\operatorname{Im} \Sigma(\mathbf{k}, \omega) & \rightarrow \operatorname{Im} \Sigma(\mathbf{k}+\mathbf{Q},-\omega), \\
A(\mathbf{k}, \omega) & \rightarrow A(\mathbf{k}+\mathbf{Q},-\omega) .
\end{aligned}
$$

The charge correlation function does not change. Figures 2, 5 , 6, 7, and 9 are transformed to those summarized in Fig. 12. Results corresponding to Figs. 3 and 4 are presented in Appendix B. Note that this kind of transformation is not necessary for h-cuprates.

\section{G. Absence of long-range Coulomb interaction}

We have considered the long-range Coulomb interaction [Eq. (9)]. What happens if we replace it with a short-range Coulomb interaction? As a typical short-range Coulomb interaction, we may consider

$$
V(\mathbf{q})=V_{1}\left(\cos q_{x}+\cos q_{y}\right)+V_{2} \cos q_{z} .
$$

The charge excitation spectrum for $V_{2}=0$ and $t_{z}=0$ was already shown in Ref. [56], where the zero-sound mode is realized as collective excitations, which are gapless at $\mathbf{q}=$ $\left(0,0, q_{z}\right)$ independent of $q_{z}$. In the present layered model with a finite $t_{z}$, the zero-sound mode acquires a gap at $\mathbf{q}=\left(0,0, q_{z}\right)$ for $q_{z} \neq 0$ but remains gapless for $q_{z}=0$; see Ref. [17] for further details. We have chosen $V_{1}=V_{2}=1$ and computed the intensity map of the one-particle spectral function.

The obtained result is shown in Fig. 13, which should be compared with Fig. 7, where the long-range Coulomb interaction is considered. The crucial differences appear in the two incoherent bands above the Fermi energy. The band around $\omega / t \approx 1$ seen in Fig. 7 is now pushed down toward the main quasiparticle band and is hardly recognized in Fig. 13. The other incoherent band around $\omega / t \approx 2$ becomes clearer than Fig. 7 (see also Fig. 5), where the corresponding band does form around $\omega / t \approx 2$ but is invisible in that intensity scale. Minor differences are also recognized. We now have a clear mustache in Fig. 13 just below the quasiparticle dispersion on both sides of $\mathbf{k}=\left(0,0, k_{z}\right)$, which comes from coupling to the zero-sound mode. The sharp side band deeply below the Fermi energy is robust. Quantitatively it is pushed up and becomes closer to the main quasiparticle band. 

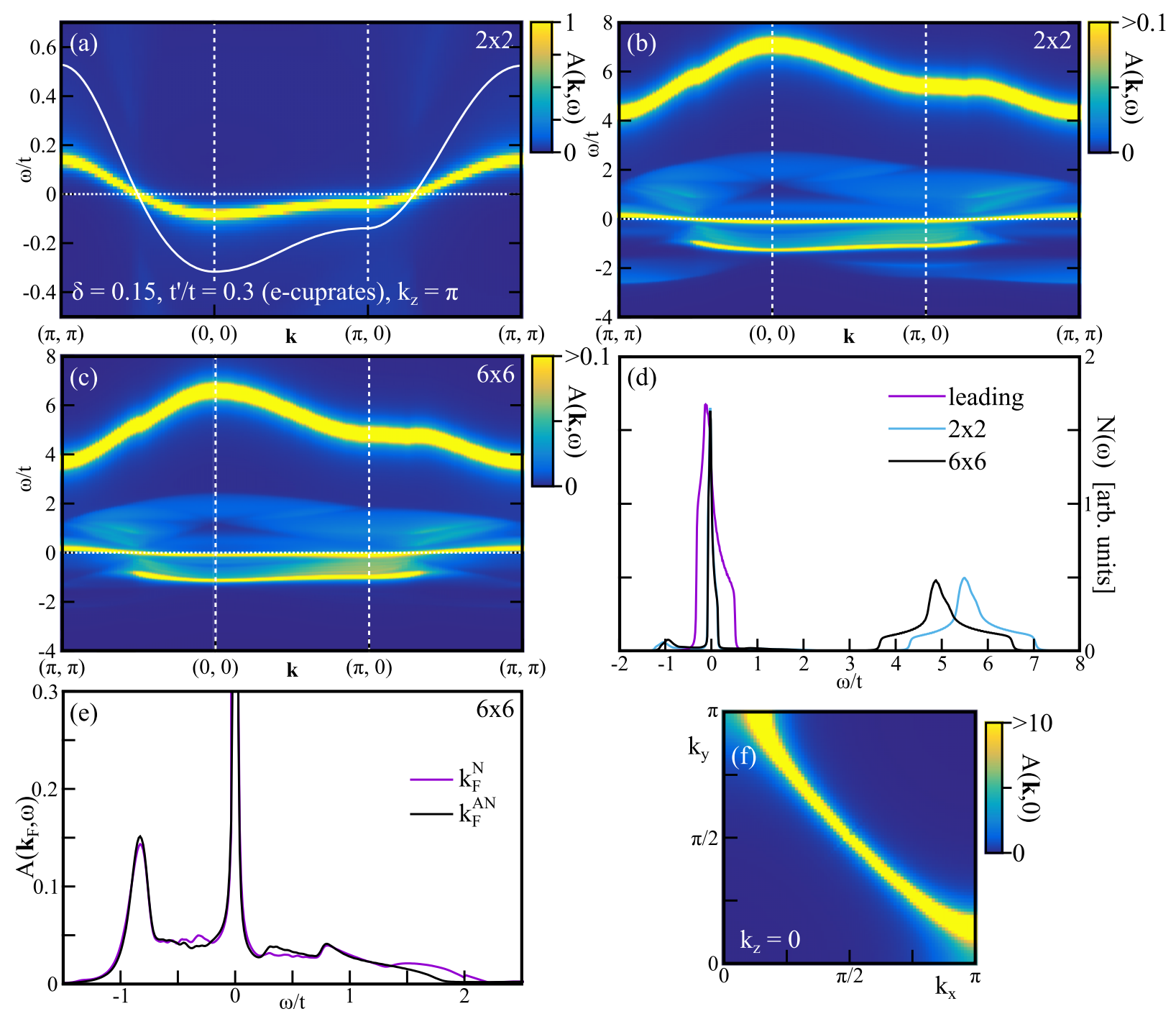

FIG. 12. Intensity map of $A(\mathbf{k}, \omega)$ along the direction $(\pi, \pi)-(0,0)-(\pi, 0)-(\pi, \pi)$ after the particle-hole transformation (a) around the Fermi energy corresponding to Fig. 2, and (b) and (c) in a larger energy region corresponding to Fig. 5 and 7, respectively. (d) The density of states after the particle-hole transformation of Fig. 6. (e) The spectral function $A\left(\mathbf{k}_{F}, \omega\right)$ at the nodal and antinodal Fermi momenta. (f) Intensity map of $A(\mathbf{k}, 0)$ at the Fermi energy in the first quadrant of the Brillouin zone. (e) and (f) are obtained after the particle-hole transformation of Figs. 9(b) and 9(c), respectively.

\section{H. $\boldsymbol{t}^{\prime}$ dependence}

The band parameter $t^{\prime}$ is often utilized to describe a material dependence in the cuprate family. In fact, the tendency to various bond-charge-order instabilities depends on the value of $t^{\prime}$ and, in particular, the sign change of $t^{\prime}$ leads to a big difference $[19,45]$. However, the effect of bond-charge fluctuations turns out to be very weak on the electron self-energy. Moreover, essential features of on-site charge fluctuations including plasmons are not altered by a different choice of $t^{\prime}$ unless one is interested in fine fitting to experimental data [29]. Hence the results obtained in the present paper do not depend much on a choice of $t^{\prime}$. This is also recognized by noting similar results for both e- and h-cuprates; see Figs. 5, 7, 10(a), and 10(b). We therefore expect that the present results manifest a generic feature of the spectral function when considering the electron self-energy from quantum charge fluctuations in the $t-J$ model.

\section{DISCUSSIONS}

We have found that the electron self-energy is controlled mainly by the on-site charge fluctuations describing plasmons. This conclusion might seem counterintuitive because there are also bond-charge fluctuations which are present in the low-energy region with the scale of $J$ and thus seem to contribute much to the self-energy especially near the Fermi energy. The point lies in the strength of the coupling between bond-charge fluctuations and electrons. The on-site charge fluctuations are described by the components $a, b=1,2$ and the bond-charge ones by $a, b=3-6$ in Eq. (14). The vertex $h_{a}(\mathbf{k}, \mathbf{q}, v)$ with $a=3-6$ is proportional to $\Delta$ [see Eq. (12)], whose typical value is around $0.025-0.03 t$. Since the contribution from the bond-charge fluctuations is proportional to $\Delta^{2}$, its effect becomes much weaker than that from the on-site charge fluctuations. 


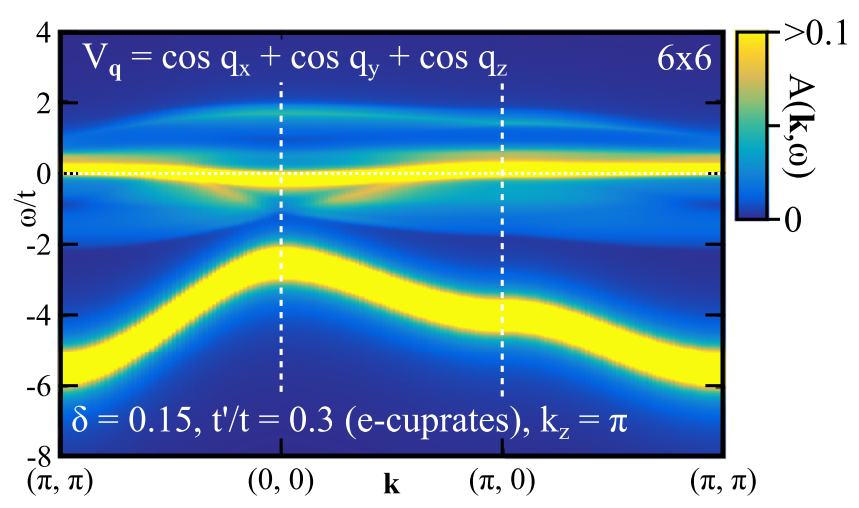

FIG. 13. Intensity map of $A(\mathbf{k}, \omega)$ in the absence of the longrange Coulomb interaction along the direction $(\pi, \pi)-(0,0)-(\pi, 0)$ $(\pi, \pi)$ in the hole picture; both on-site charge and bond-charge fluctuations are taken into account. $k_{z}$ dependence is weak and $k_{z}=$ $\pi$ is taken as a representative value. The corresponding result in the particle picture is presented in Appendix B.

The factor of $\Delta^{2}$ can be generic to strongly correlated electron systems. In fact, similar features are recognized in slave-boson calculations [46], the Kadanoff-Baym method for Hubbard operators [68], the equation of motion method for the Green's functions in terms of the Hubbard operators [69], and the equations of motion for projected operators on the $t-J$ model [70].

The results for e-cuprates [Figs. 12(b) and 12(c)] look different from those for h-cuprates [Figs. 10(a) and 10(b)] in a usual particle picture. However, when we take a hole picture for e-cuprates (Figs. 5 and 7), those become essentially the same. This is because the obtained self-energy and the resulting one-particle spectral function are determined mainly by the coupling to the on-site charge fluctuations, which are almost the same for both e- and h-cuprates in the present theory [17]. The major difference of charge excitations between e- and h-cuprates appears in the bond-charge fluctuations [45], whose contribution to the self-energy is, however, minor even close to bond-charge instabilities at $T=0$. Given that the onsite charge excitations obtained in the present theory capture the experimental data in both e- and h-cuprates [16-18,29], the present paper implies that the obtained one-particle excitation spectra are universal in cuprates.

We have shown that the coupling to plasmon excitations generates an incoherent band around $1 t$ in Figs. 10(a) and 10(b). This emergent band can also be seen as the density of state generated in the corresponding energy region in Fig. 6. In fact, early studies reported the peak around $1 \mathrm{eV}$ above the Fermi energy by the inverse photoemission spectroscopy for h-cuprates [71,72]. The reported state can be our incoherent band generated by the coupling to plasmons since we can reasonably assume $t / 2 \approx 500 \mathrm{meV}$ for cuprates (Refs. [73,74]). The spectral weight of our incoherent band is enhanced along the direction $(\pi, 0)-(\pi, \pi)-(\pi / 2, \pi / 2)$, which can be tested by the inverse ARPES. On the other hand, for e-cuprates, this incoherent band emerges below the Fermi energy along the direction $(\pi / 2, \pi / 2)-(0,0)-(\pi, 0)$ as shown in Figs. 12(b) and $12(\mathrm{c})$ (in the usual particle picture). Given that plasmon excitations are now observed in experiments [11,12,28,29], it is interesting to test the predicted band by performing ARPES for e-cuprates.

This test is also important to highlight the role of the long-range Coulomb interaction in cuprates. Traditionally, short-range electron-electron interactions are presumed in most studies on cuprates. In fact, there have been experimental reports recently which are in disfavor with plasmon excitations [30,31]. In this context, our results without the long-range Coulomb interaction (Figs. 13 and 17) are useful. They confirm that the emergent incoherent band around $|\omega / t| \approx 1$ [Figs. 10(a), 10(b), 12(b), and 12(c)] indeed comes from the coupling to plasmons, namely, through the longrange Coulomb interaction.

The on-site charge fluctuations also generate a band around $5 t$ for e-cuprates [Figs. 12(b) and 12(c)] and around $-5 t$ for h-cuprates [Figs. 10(a) and 10(b)]. A very similar feature was also obtained in the two-mode variational Monte Carlo study of the $t-J$ model [75]. It may not be easy to test this emergent band experimentally since the distinction from additional bands, not included in the present one-band $t-J$ model, is not straightforward in real materials.

As shown in Fig. 8, the quasiparticle weight $Z$ is suppressed dramatically by on-site charge fluctuations and becomes smaller with decreasing carrier density, although charge fluctuations themselves are driven by the doped carrier. The similar doping dependence of $Z$ was also obtained in the multiband Hubbard model in the local density-approximation in combination with the dynamical mean-field theory [76]. The present paper suggests that charge fluctuations are the major contribution to yield the suppression of the quasiparticle weight in the doped Mott insulator even though magnetic fluctuations are expected strong near half filling.

It is well known that one of the most important issues in cuprates is the pseudogap phase [1], which is clearly resolved in h-cuprates, while its existence is controversial in e-cuprates [77]. As seen in Figs. 6, 9, and 11, the quantum charge fluctuations do not produce a pseudogap feature, although the spectral width in Figs. 9(c) and 11(b) becomes sharpest at the nodal region and broadest at the antinodal region, which somewhat shares a $d$-wave feature, the same symmetry of the pseudogap. It seems too hasty to infer that the pseudogap may originate from some other degree of freedom such as spin fluctuations. In fact, our calculations have been performed at $T=0$ and thus the charge fluctuations we have studied are purely quantum. At finite temperatures, classical fluctuations also contribute to the self-energy [78,79]. We leave this possibility to a future issue.

A renormalization of the electron dispersion is related directly to the so-called kinks of the dispersion observed by ARPES. Mainly two different kinks are reported: lowenergy (40-70 meV) (Refs. [38,80-86]) and high-energy ( $\sim 0.3 \mathrm{eV})$ (Refs. [34-39]) kinks. The low-energy kink was discussed in terms of a coupling to magnetic resonance mode $[80,82,83,85,87,88]$, spin fluctuations $[86,89]$, and phonons $[81,84,88,90]$, and also even without invoking such a coupling to bosons [91], whereas the high-energy kink may come from spin fluctuations [92,93], incoherent hole motion [33], string 
excitations [94], and some extrinsic effects [95]. Although we have performed a comprehensive analysis of the electron self-energy from all possible charge fluctuations included in the $t-J$ model at the order of $1 / N$, we did not obtain a clear kink structure associated with experimental data if we assume $t / 2 \approx 500 \mathrm{meV}$.

The fact that we did not obtain a clear kink might seem peculiar because it is well recognized that the electron dispersion may exhibit a kink near a typical energy scale of bosonic fluctuations via their coupling to electrons. The reasons are twofold: energy scale and coupling strength. As shown in Fig. 2, the quasiparticle dispersion is strongly renormalized by the coupling to charge fluctuations and is realized in $-0.2 \lesssim$ $\omega / t \lesssim 0.1$. In such a low energy scale, it is only bond-charge fluctuations which could play a role because on-site charge fluctuations have energy much larger than the renormalized dispersion. However, as we have shown and discussed, the effect of bond-charge fluctuations is substantially weakened by the factor of $\Delta^{2}$ coming from the vertex $h_{a}(\mathbf{k}, \mathbf{q}, v)$ [see Eq. (12)] and cannot generate a clear kink in the electron dispersion.

Our results shown in Figs. 5, 7, and 10(a) and 10(b) indicate a large spectral weight around $\omega \sim-4 t$ near $\mathbf{k}=$ $\left(0,0, k_{z}\right)$, which was also obtained in finite-temperature diagonalization of small clusters [33]. Although Ref. [33] discussed the high-energy kink [34-39] on the basis of such results, we do not because the absolute energy becomes different by more than a factor of 4 if we assume $t / 2 \approx 500 \mathrm{meV}$. Considering that our charge excitation spectra capture the experimental data, including quantitative aspects in many cases $[14,15,17,18,29]$, we think that a factor of 4 is too large as the energy difference. We hope that this different view from Ref. [33] stimulates a further study of the high-energy kink, whose origin remains controversial and will lead to a better understanding of the nature of the self-energy in cuprates.

Instead of a kink in the electron dispersion, we predict a cascadelike feature in the sense that the spectral weight of the quasiparticle dispersion flows to the incoherent band around $\omega / t \sim-1$, especially in wide regions around $\mathbf{k} \approx$ $\left(\pi, 0, k_{z}\right)$ and $\left(\pi / 2, \pi / 2, k_{z}\right)$ for e-cuprates in Figs. 12(b) and 12(c). This feature should not be confused with the "waterfall" reported around $-(0.3 \sim 1) \mathrm{eV}$ and $\mathbf{k} \approx\left(0,0, k_{z}\right)$ for $\mathrm{h}$-cuprates [34-39]. We did not obtain such a waterfall clearly for h-cuprates; see Figs. 10(a) and 10(b).

The effect of plasmons on the electron dispersion in $\mathrm{h}$ cuprates was studied in Ref. [32] in a phenomenological scheme. They reported that a coupling to plasmons generates shadow bands around $1.5 \mathrm{eV}$ below and above the LDA band. This qualitative feature is shared with our results in that we have also obtained bands generated around the energy $-5 t$ and $1 t$ in Figs. 10(a) and 10(b). Furthermore, Ref. [32] concluded that plasmons cannot explain the high-energy kink of the electron dispersion around $-(0.3 \sim$ 1) eV (Refs. [34-39]), but the plasmons strongly renormalize the electron dispersion. These insights are also shared with ours obtained in the present microscopic analysis of the selfenergy.

Although we do not share a view of the high-energy kink with Ref. [33], the present work is complementary to
Ref. [33]. Reference [33] performed numerical analysis by using small clusters of the $t-J$ model, but did not consider the effect of plasmons. On the other hand, we focus on the charge sector and analyze all fluctuations including plasmons in the thermodynamic limit. Since both magnetic and charge fluctuations were involved in Ref. [33], it remained unclear what kind of fluctuations were actually relevant to the spectral function. The present paper then clarifies that strong electron correlations due to the on-site charge fluctuations including plasmons, not bond-charge nor magnetic ones, play a dominant role to form the electron spectral function. Reference [33] obtained the imaginary part of the electron self-energy with strong particle-hole asymmetry. This asymmetry was understood as a consequence from the strong correlation effects due to the local constraint in the $t-J$ model in Ref. [33]. This insight fully agrees with the present paper as we explain in the context of Fig. 3. While Ref. [33] did not consider the long-range Coulomb interaction, we find that it leads to a side band around $|\omega / t| \approx 1$. In addition, we elucidate the quasiparticle weight, its doping dependence, and the interplay of on-site charge and bond-charge fluctuations.

Reference [96] studied the spectral function in the chargestripe phase. While no tendency toward charge stripes was obtained in the present theory $[19,45]$, a certain bond-charge order has the same symmetry as stripes [19]. In this sense, we might mimic the effect of stripes on the spectral function and make an interesting comparison with Ref. [96]. However, as we have showed in the present paper, the effect of bond-charge fluctuations is minor on the electron self-energy.

We have employed a layered model to perform a realistic analysis of the self-energy from the charge excitations and considered the long-range Coulomb interaction in threedimensional space [see Eq. (9)]. This three-dimensional Coulomb interaction is important to take a plasmon contribution into account appropriately. Concerning the electron band structure, however, we find that essentially the same results are obtained for the electron self-energy even if we set $t_{z}=0$. In this sense, the detail of three-dimensionality of the band is not crucial to the electron self-energy from charge fluctuations, at least at zero temperature.

The oxygen degrees of freedom in cuprates are included effectively in the one-band $t-J$ model as the Zhang-Rice singlet [50] and charge fluctuations at the oxygen sites are described as bond-charge fluctuations in the present theory. However, this does not mean that the effect of oxygens is included perfectly in the $t-J$ model. For example, if we wish to address the issue why $\mathrm{Cu}$ L-edge RIXS detects the plasmons in e-cuprates efficiently $[2,7,8,10-12]$ while it does not in h-cuprates [27,29], we feel that the $t-J$ model is not enough and the oxygen degrees of freedom should be considered on an equal footing.

Finally, it is natural to ask whether our obtained results could stay if one studies the electron self-energy beyond the present approximation at the order of $1 / N$. While this possibility is not easily addressed, we believe that the essential features may not be modified much on the basis of a comparison with numerical analysis of the $t-J$ (Refs. [33,75]) and Hubbard [76] models as we have discussed above. 


\section{CONCLUDING REMARKS}

The electron self-energy in cuprates was mainly studied by considering a coupling to spin fluctuations $[80,82,83,85-$ $89,92,93]$ and phonons $[81,84,88,90]$. Compared with those studies, the effect of charge fluctuations on the electron selfenergy has been studied much less $[32,33]$. Given that high- $T_{c}$ superconductivity occurs upon doping of charged carriers [1] and the charge dynamics was revealed in $(\mathbf{q}, \omega)$ space recently [2-12,27-29], it is challenging to clarify how the actual charge dynamics impacts the one-particle properties, which will then deepen our understanding of the cuprate phenomenology. In this paper, we have performed a comprehensive analysis of the electron self-energy from quantum charge fluctuations in a realistic layered $t-J$ model, which can capture the charge excitation spectra observed in cuprates. We have found that on-site charge fluctuations have a great impact on the electron self-energy. The quasiparticle weight is reduced significantly, which occurs almost isotropically along the Fermi surface. In addition, mainly two additional bands emerge. In particular, the emergent band around $\omega / t \approx 1$ with strong intensity along $(\pi, 0)-(\pi, \pi)-(\pi / 2, \pi / 2)$ in Figs. 5, 7 , and 10 [see also Figs. 12(b) and 12(c) where it occurs around $\omega / t \approx-1$ along $(\pi / 2, \pi / 2)-(0,0)-(\pi, 0)]$ comes from the coupling to the plasmons [16-18] recently identified by x-ray measurements $[11,12,28,29]$. Surprisingly, the effect of lowenergy bond-charge quantum fluctuations is minor even close to bond-charge instabilities and the electron self-energy is mainly controlled by the on-site charge fluctuations describing plasmons. Furthermore, the quantum charge dynamics does not produce a clear kink nor a pseudogap feature in the electron dispersion.

\section{ACKNOWLEDGMENTS}

The authors thank M. Hepting, P. Horsch, A. Oleś, and R. Zeyher for fruitful comments. A.G. thanks the Max-PlanckInstitute for Solid State Research in Stuttgart for hospitality and financial support. H.Y. was supported by JSPS KAKENHI Grants No. JP18K18744 and No. JP20H01856.

\section{APPENDIX A: COMPLETE FORMALISM}

We start with the layered $t-J$ model with the long-range Coulomb interaction:

$$
\begin{aligned}
H= & -\sum_{i, j, \sigma} t_{i j} \tilde{c}_{i \sigma}^{\dagger} \tilde{c}_{j \sigma}+\sum_{\langle i, j\rangle} J_{i j}\left(\vec{S}_{i} \cdot \vec{S}_{j}-\frac{1}{4} n_{i} n_{j}\right) \\
& +\frac{1}{2} \sum_{i, j} V_{i j} n_{i} n_{j} .
\end{aligned}
$$

This model is defined in the restricted Hilbert space, where the double occupancy of electrons is prohibited at any lattice site. We can write the $t-J$ model in terms of nine Hubbard $\hat{X}$ operators [97]: $\tilde{c}_{i \sigma}^{\dagger}=\hat{X}_{i}^{\sigma 0}, \tilde{c}_{i \sigma}=\hat{X}_{i}^{0 \sigma}, S_{i}^{+}=\hat{X}_{i}^{\uparrow \downarrow}, S_{i}^{-}=\hat{X}_{i}^{\downarrow \uparrow}$, $n_{i}=\hat{X}_{i}^{\uparrow \uparrow}+\hat{X}_{i}^{\downarrow \downarrow}$, and $\hat{X}_{i}^{00}$ describing the number of doped holes; the $z$ component of the spin operator is described by $S_{i}^{z}=\frac{1}{2}\left(\hat{X}_{i}^{\uparrow \uparrow}-\hat{X}_{i}^{\downarrow \downarrow}\right)$. The operators $\hat{X}_{i}^{\sigma 0}$ and $\hat{X}_{i}^{0 \sigma}$ are called fermionlike, whereas the operators $\hat{X}_{i}^{\sigma \sigma^{\prime}}$ and $\hat{X}_{i}^{00}$ are bosonlike. After introducing the chemical potential $\mu$, the resulting
Hamiltonian becomes

$$
\begin{aligned}
H(X)= & -\sum_{i, j, \sigma} t_{i j} \hat{X}_{i}^{\sigma 0} \hat{X}_{j}^{0 \sigma} \\
& +\frac{J}{2} \sum_{\langle i, j\rangle, \sigma \sigma^{\prime}}\left(\hat{X}_{i}^{\sigma \sigma^{\prime}} \hat{X}_{j}^{\sigma^{\prime} \sigma}-\hat{X}_{i}^{\sigma \sigma} \hat{X}_{j}^{\sigma^{\prime} \sigma^{\prime}}\right) \\
& +\frac{1}{2} \sum_{i, j, \sigma \sigma^{\prime}} V_{i j} \hat{X}_{i}^{\sigma \sigma} \hat{X}_{j}^{\sigma^{\prime} \sigma^{\prime}}-\mu \sum_{i, \sigma} \hat{X}_{i}^{\sigma \sigma} .
\end{aligned}
$$

There are two major difficulties in the Hamiltonian Eq. (A2): the complicated commutation rules of the Hubbard operators [97] and the absence of a small parameter. A popular method to handle the former difficulty is to use slave particles. For instance, in the slave-boson method [52], the original fermionlike $\hat{X}^{0 \sigma}$ operator is written as $\hat{X}^{0 \sigma}=\hat{b}^{\dagger} \hat{f}_{\sigma}$, where $\hat{b}$ and $\hat{f}_{\sigma}$ are usual boson and fermion operators, respectively. This scheme introduces a gauge field, which requires a gauge fixing and the introduction of a Faddeev-Popov determinant [98]. Gauge fluctuations should be taken into account beyond mean-field theory and the slave particles need to be convoluted to form the original fermionic operator $\tilde{c}$.

An alternative approach is to employ the Faddeev-Jackiw [99] and Dirac [100,101] theories for constrained systems and to develop a path integral representation for the Hubbard operators [102]. The partition function $Z$ is given in the Euclidean form as follows:

$$
\begin{aligned}
Z= & \int \mathcal{D} X_{i}^{\alpha \beta} \delta\left(X_{i}^{00}+\sum_{\sigma} X_{i}^{\sigma \sigma}-1\right) \delta\left(X_{i}^{\sigma \sigma^{\prime}}-\frac{X_{i}^{\sigma 0} X_{i}^{0 \sigma^{\prime}}}{X_{i}^{00}}\right) \\
& \times\left(\operatorname{sdet} M_{A B}\right)_{i}^{\frac{1}{2}} \exp \left(-\int d \tau L_{E}(X, \dot{X})\right),
\end{aligned}
$$

where fermionlike and bosonlike $\mathrm{X}$-operators are described by Grassmann and usual complex variables, respectively, $\tau=i t$ and $\dot{X}=\partial_{\tau} X$. Note that we have removed the hat symbol of the Hubbard operators because they become classical fields in the path integral formulation. The Euclidean Lagrangian $L_{E}(X, \dot{X})$ is given by

$$
L_{E}(X, \dot{X})=\frac{1}{2} \sum_{i, \sigma} \frac{\left(\dot{X}_{i}^{0 \sigma} X_{i}^{\sigma 0}+\dot{X}_{i}^{\sigma 0} X_{i}^{0 \sigma}\right)}{X_{i}^{00}}+H(X) .
$$

The superdeterminant

$$
\left(\operatorname{sdet} M_{A B}\right)_{i}^{\frac{1}{2}}=\left(-X_{i}^{00}\right)^{2}
$$

is equivalent to the determinant of the Dirac matrix $[100,101]$ formed by the constraints and also to the Faddeev-Jackiw theory, where the symplectic matrix has even and odd Grassmann elements (see Ref. [103] and references therein). The two constraints specified by the two $\delta$-functions in Eq. (A3) are necessary to recover the correct algebra of the original Hubbard operators [102]. The method used here recovers [103] the coherent state path-integral representation for the $t-J$ model [104].

The partition function Eq. (A3) looks different from that usually found in other solid state problems. The measure of the integral contains the two constraints as well as a superdeterminant $\left(\operatorname{sdet} M_{A B}\right)_{i}^{\frac{1}{2}}$. In addition, the kinetic term of the Lagrangian Eq. (A4) is nonpolynomial. As a result, even 
if we discard the Heisenberg interaction and the long-range Coulomb interaction in the $t-J$ model, the theory is still highly nontrivial. In fact, the electron-electron interactions also come from the algebra of the $\mathrm{X}$ operators in the $t-J$ model.

One of the important aspects of the $t-J$ model is that charge carriers are absent at half-filling. To describe this explicitly, we rewrite the terms $X_{i}^{\sigma \sigma} X_{j}^{\sigma^{\prime} \sigma^{\prime}}$ in Eq. (A2) in terms of $X_{i(j)}^{00}$ by using the local constraint $X_{i(j)}^{00}+\sum_{\sigma} X_{i(j)}^{\sigma \sigma}=1$. This local constraint is then kept by introducing the Lagrange multiplier $\lambda_{i}$ :

$$
\begin{aligned}
& \delta\left(X_{i}^{00}+\sum_{\sigma} X_{i}^{\sigma \sigma}-1\right) \\
& =\int \mathcal{D} \lambda_{i} \exp \left(i \lambda_{i}\left(X_{i}^{00}+\sum_{\sigma} X_{i}^{\sigma \sigma}-1\right)\right) .
\end{aligned}
$$

The remaining two terms, $X_{i}^{\sigma \sigma^{\prime}} X_{j}^{\sigma^{\prime} \sigma}$ in the $J$ term and the term of the chemical potential, are replaced by integrating out the bosonic variable $X^{\sigma \sigma^{\prime}}$ using the second $\delta$-function in Eq. (A3), i.e., $X_{i}^{\sigma \sigma^{\prime}}=\left(X_{i}^{\sigma 0} X_{i}^{0 \sigma^{\prime}}\right) / X_{i}^{00}$. The resulting model is then described by $X_{i}^{\sigma 0}, X_{i}^{0 \sigma}, X_{i}^{00}$, and $\lambda_{i}$, and their mutual interactions.

To achieve the systematic analysis of the $t$ - $J$ model, we employ a nonperturbative technique based on a large- $N$ expansion [44], where $N$ is the number of electronic degrees of freedom per site and $1 / N$ is assumed to be a small parameter. We then extend the spin index $\sigma$ to a new index $p$ running from 1 to $N$. To get a finite theory in the limit of $N \rightarrow \infty$, we rescale the hopping $t_{i j}$ to $t_{i j} / N, J$ to $J / N$, and $V_{i j}$ to $V_{i j} / N$. The completeness condition, i.e., the local constraint, becomes the $N$-extended one: $X_{i}^{00}+\sum_{p} X_{i}^{p p}=N / 2$. From this completeness condition, we can see that $X^{00}$ is $O(N)$ and $X^{p p}$ is $O(1)$. As a consequence of this, the large- $N$ approach weakens the effective spin interaction compared with the one associated with the charge degrees of freedom. In this sense, the large- $N$ scheme is potentially interesting in light of $\mathrm{x}$-ray experiments [2-12,27-29] which show unexpectedly strong excitations in the pure charge-channel.

We write the boson fields $X_{i}^{00}$ and $\lambda_{i}$ in terms of static mean-field values, $\left(r_{0}, \lambda_{0}\right)$, and dynamic fluctuations, $\left(\delta R_{i}, \delta \lambda_{i}\right)$ :

$$
\begin{aligned}
X_{i}^{00} & =N r_{0}\left(1+\delta R_{i}\right), \\
\lambda_{i} & =\lambda_{0}+\delta \lambda_{i} .
\end{aligned}
$$

For the fermionlike fields, we write as follows:

$$
\begin{aligned}
f_{i p}^{+} & =\frac{1}{\sqrt{N r_{0}}} X_{i}^{p 0}, \\
f_{i p} & =\frac{1}{\sqrt{N r_{0}}} X_{i}^{0 p} .
\end{aligned}
$$

Using Eq. (A7) and the completeness condition, we obtain $r_{0}=\delta / 2$, where $\delta$ is the hole doping rate away from half filling. The fermion variable $f_{i p}$ is proportional to $X_{i}^{0 p}$ and should not be associated with the so-called spinon in the slave-boson method.

$X^{00}$ to the second power appears in Eq. (A5) because we were working with two spin projections. After the extension to large- $N$, the superdeterminant becomes

$$
\left(\operatorname{sdet} M_{A B}\right)_{i}^{\frac{1}{2}}=\left(N r_{0}\right)^{N}\left[-\left(1+\delta R_{i}\right)\right]^{N} .
$$

The constant factor $\left(N r_{0}\right)^{N}$ contributes to the path-integral normalization factor. On the other hand, the term $[-(1+$ $\left.\left.\delta R_{i}\right)\right]^{N}$ can be written in terms of $N$ complex bosonic ghost fields $\mathcal{Z}_{i p}$ in an integral representation (Ref. [44]). Therefore the superdeterminant contributes to the effective Lagrangian as

$$
L_{\text {ghost }}(\mathcal{Z})=-\sum_{i p} \mathcal{Z}_{i p}^{\dagger}\left(\frac{1}{1+\delta R_{i}}\right) \mathcal{Z}_{i p} .
$$

The exchange interaction term $(J$ term) contains the four fermion fields and the two bosons in the denominator. They can be decoupled through a Hubbard-Stratonovich transformation by introducing the field $\Delta_{i j}$,

$$
\Delta_{i j}=\frac{J}{4 N} \sum_{p} \frac{f_{j p}^{\dagger} f_{i p}}{\sqrt{\left(1+\delta R_{i}\right)\left(1+\delta R_{j}\right)}} .
$$

This field describes bond-charge fluctuations because the fermions with the same spin projection $p$ sit on the nearestneighbor sites and the sum over $p$ is taken. This bond field $\Delta_{i j}$ is parameterized as

$$
\Delta_{i}^{\eta}=\Delta\left(1+r_{i}^{\eta}+i A_{i}^{\eta}\right),
$$

where $r_{i}^{\eta}$ and $A_{i}^{\eta}$ correspond to the real and imaginary parts of the bond-field fluctuations, respectively, and $\Delta$ is a static mean-field value. The index $\eta$ denotes the bond directions $\eta_{1}=(1,0)$ and $\eta_{2}=(0,1)$ on a square lattice. Finally, we expand the term $1 /(1+\delta R)$ in powers of $\delta R$, which generates various interactions between fermions and bosons. The number of the interactions considered in theory is controlled in powers of $1 / N$.

We may define a six-component boson field

$$
\delta X^{a}=\left(\delta R, \delta \lambda, r^{\eta_{1}}, r^{\eta_{2}}, A^{\eta_{1}}, A^{\eta_{2}}\right),
$$

where the first component $\delta R$ is related to on-site charge fluctuations and $\delta \lambda$ is the fluctuation of the Lagrange multiplier $\lambda_{i}$ associated with the completeness condition; the remaining components come from fluctuations of the bond field [see Eq. (A14)]. Hence the effective theory can be described in terms of boson fields $\delta X^{a}$, fermion fields $f_{i p}$, ghost fields $\mathcal{Z}_{i p}$, and their mutual interactions.

From the quadratic part for fermions we obtain the bare electron propagator in the paramagnetic phase (solid line in Fig. 14):

$$
G_{p p}^{(0)}\left(\mathbf{k}, \mathrm{i} \omega_{n}\right)=\frac{1}{\mathrm{i} \omega_{n}-\varepsilon_{\mathbf{k}}} .
$$

Here $\mathbf{k}$ and $i \omega_{n}$ are momentum and fermionic Matsubara frequency, respectively. The bare fermionic propagator $G^{(0)}$ is $O(1)$. The electron dispersion $\varepsilon_{\mathbf{k}}$ is written as

$$
\varepsilon_{\mathbf{k}}=\varepsilon_{\mathbf{k}}^{\|}+\varepsilon_{\mathbf{k}}^{\perp},
$$

where the in-plane dispersion $\varepsilon_{\mathbf{k}}^{\|}$and the out-of-plane disper$\operatorname{sion} \varepsilon_{\mathbf{k}}^{\perp}$ are given, respectively, by

$$
\varepsilon_{\mathbf{k}}^{\|}=-2\left(t \frac{\delta}{2}+\Delta\right)\left(\cos k_{x}+\cos k_{y}\right)-4 t^{\prime} \frac{\delta}{2} \cos k_{x} \cos k_{y}-\mu,
$$

$$
\varepsilon_{\mathbf{k}}^{\perp}=-2 t_{z} \frac{\delta}{2}\left(\cos k_{x}-\cos k_{y}\right)^{2} \cos k_{z} .
$$




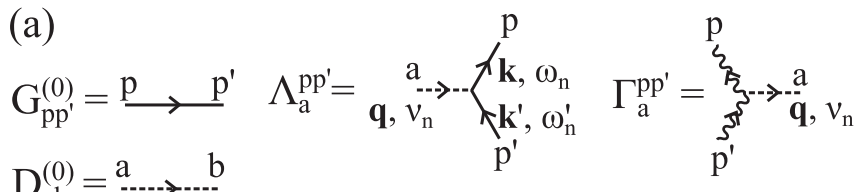

$\mathrm{D}_{\mathrm{ab}}^{(0)}=\stackrel{\mathrm{a}}{\cdots-\cdots} \mathrm{b}$

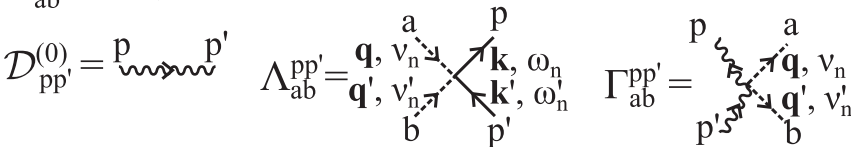

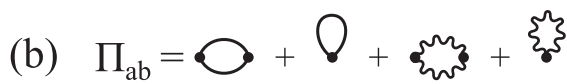

$\mathrm{D}_{\mathrm{ab}}^{-1}=(=:=: \Rightarrow \mathrm{z}=:=:)^{-1}=\left[\mathrm{D}_{\mathrm{ab}}^{(0)}\right]^{-1}-\Pi_{\mathrm{ab}}$

(c) $\Sigma=\Sigma^{(1)}+\Sigma^{(2)}=$

FIG. 14. (a) Propagators and vertices in a large- $N$ theory up to $O(1 / N)$. Solid line represents the fermion propagator $G_{p p^{\prime}}^{(0)}$; dashed line the $6 \times 6$ boson propagator $D_{a b}^{(0)}$ for the six-component fields $\delta X^{a}$; wavy line the ghost propagator $\mathcal{D}_{p p^{\prime}}^{(0)} . \Lambda_{a}^{p p^{\prime}}$ and $\Gamma_{a}^{p p^{\prime}}$ describe the three-point interaction between two fermions with spin indices $p$ and $p^{\prime}$ and one boson with the component $a$, and two ghost fields with one boson, respectively. $\Lambda_{a b}^{p p^{\prime}}$ and $\Gamma_{a b}^{p p^{\prime}}$ are the four-point interactions among the two fermions and two bosons, and two ghost fields and two bosons, respectively. (b) The irreducible boson self-energy $\Pi_{a b}$ and the renormalized boson propagator $D_{a b}$. (c) The electron selfenergy $\Sigma(\mathbf{k}, \omega)$ at the order of $1 / N$.

Here $\lambda_{0}$ in Eq. (A8) was absorbed in the chemical potential $\mu$. In-plane momenta $k_{x}$ and $k_{y}$ and out-of-plane momentum $k_{z}$ are measured in units of $a^{-1}$ and $d^{-1}$, respectively; $a$ is the lattice constant in the square lattice and $d$ is the distance between the planes. The quantity $\Delta$ is given by the expression

$$
\Delta=\frac{J}{4 N_{s} N_{z}} \sum_{\mathbf{k}}\left(\cos k_{x}+\cos k_{y}\right) n_{F}\left(\varepsilon_{\mathbf{k}}\right)
$$

where $n_{F}$ is the Fermi function, $N_{s}$ is the total number of lattice sites on the square lattice, and $N_{z}$ is the number of layers along the $z$ direction. For a given doping $\delta, \mu$, and $\Delta$ are determined self-consistently by solving Eq. (A20) and

$$
(1-\delta)=\frac{2}{N_{s} N_{z}} \sum_{\mathbf{k}} n_{F}\left(\varepsilon_{\mathbf{k}}\right) .
$$

From the quadratic part of the six-component boson field $\delta X^{a}$, we obtain the inverse of the bare bosonic propagator (dashed line in Fig. 14), connecting two components $a$ and $b$,

$$
\begin{aligned}
& {\left[D_{a b}^{(0)}\left(\mathbf{q}, i v_{n}\right)\right]^{-1}} \\
& \quad=N\left(\begin{array}{cccccc}
\frac{\delta^{2}}{2}[V(\mathbf{q})-J(\mathbf{q})] & r_{0} & 0 & 0 & 0 & 0 \\
r_{0} & 0 & 0 & 0 & 0 & 0 \\
0 & 0 & \frac{4}{J} \Delta^{2} & 0 & 0 & 0 \\
0 & 0 & 0 & \frac{4}{J} \Delta^{2} & 0 & 0 \\
0 & 0 & 0 & 0 & \frac{4}{J} \Delta^{2} & 0 \\
0 & 0 & 0 & 0 & 0 & \frac{4}{J} \Delta^{2}
\end{array}\right),
\end{aligned}
$$

where $D_{a b}^{(0)}$ is $O(1 / N), \mathbf{q}$ a three-dimensional wave vector, $v_{n}$ a bosonic Matsubara frequency, and $J(\mathbf{q})=\frac{J}{2}\left(\cos q_{x}+\cos q_{y}\right)$. $V(\mathbf{q})$ is the long-range Coulomb interaction in momentum space for a layered system

$$
V(\mathbf{q})=\frac{V_{c}}{A\left(q_{x}, q_{y}\right)-\cos q_{z}},
$$

where $V_{c}=e^{2} d\left(2 \epsilon_{\perp} a^{2}\right)^{-1}$ and

$$
A\left(q_{x}, q_{y}\right)=\alpha\left(2-\cos q_{x}-\cos q_{y}\right)+1 .
$$

These expressions are easily obtained by solving Poisson's equation on the lattice [49]. Here $\alpha=\frac{\tilde{\epsilon}}{(a / d)^{2}}, \tilde{\epsilon}=\epsilon_{\|} / \epsilon_{\perp}$, and $\epsilon_{\|}$and $\epsilon_{\perp}$ are the dielectric constants parallel and perpendicular to the planes, respectively; $e$ is the electric charge of electrons.

From the quadratic part of the ghost fields $\mathcal{Z}_{i p}$, we obtain the inverse of the bare ghost propagator (wavy line in Fig. 14),

$$
\mathcal{D}_{p p^{\prime}}^{(0)}=-\delta_{p p^{\prime}},
$$

which is $O(1)$.

The interactions between $\delta X^{a}, f_{p}$, and $\mathcal{Z}_{p}$ are given by three- and four-point vertices. The three-point vertices read

$$
\begin{aligned}
\Lambda_{a}^{p p^{\prime}}= & (-1)\left(\frac{i}{2}\left(\omega_{n}+\omega_{n}^{\prime}\right)+\mu+2 \Delta\left[\cos \left(k_{x}-\frac{q_{x}}{2}\right) \cos \frac{q_{x}}{2}+\cos \left(k_{y}-\frac{q_{y}}{2}\right) \cos \frac{q_{y}}{2}\right] ; 1 ;\right. \\
& \left.-2 \Delta \cos \left(k_{x}-\frac{q_{x}}{2}\right) ;-2 \Delta \cos \left(k_{y}-\frac{q_{y}}{2}\right) ; 2 \Delta \sin \left(k_{x}-\frac{q_{x}}{2}\right) ; 2 \Delta \sin \left(k_{y}-\frac{q_{y}}{2}\right)\right) \delta^{p p^{\prime}},
\end{aligned}
$$

and

$$
\Gamma_{a}^{p p^{\prime}}=(-1) \delta_{a 1} \delta_{p p^{\prime}}
$$

They represent the interaction between two fermions and one boson, and two ghosts and one boson, respectively. The fourpoint vertices, $\Lambda_{a b}^{p p^{\prime}}$ and $\Gamma_{a b}^{p p^{\prime}}$, describe the interaction between two fermions and two bosons, and two bosons and two ghosts, respectively. $\Lambda_{a b}^{p p^{\prime}}$ fulfills the symmetry of $\Lambda_{a b}^{p p^{\prime}}=\Lambda_{b a}^{p p^{\prime}}$. The elements different from zero are

$$
\begin{aligned}
\Lambda_{\delta R \delta R}^{p p^{\prime}}= & \left(\frac{i}{2}\left(\omega_{n}+\omega_{n}^{\prime}\right)+\mu\right. \\
& +\Delta \sum_{\eta=x, y} \cos \left(k_{\eta}-\frac{q_{\eta}+q_{\eta}^{\prime}}{2}\right) \\
& \left.\times\left(\cos \frac{q_{\eta}}{2} \cos \frac{q_{\eta}^{\prime}}{2}+\cos \frac{q_{\eta}+q_{\eta}^{\prime}}{2}\right)\right) \delta^{p p^{\prime}}
\end{aligned}
$$




$$
\begin{gathered}
\Lambda_{\delta R \delta \lambda}^{p p^{\prime}}=\frac{1}{2} \delta^{p p^{\prime}} \\
\Lambda_{\delta R r^{\eta}}^{p p^{\prime}}=-\Delta \cos \left(k_{\eta}-\frac{q_{\eta}+q_{\eta}^{\prime}}{2}\right) \cos \frac{q_{\eta}^{\prime}}{2} \delta^{p p^{\prime}},
\end{gathered}
$$

and

$$
\Lambda_{\delta R A^{\eta}}^{p p^{\prime}}=\Delta \sin \left(k_{\eta}-\frac{q_{\eta}+q_{\eta}^{\prime}}{2}\right) \cos \frac{q_{\eta}^{\prime}}{2} \delta^{p p^{\prime}} .
$$

The four-leg interaction vertex $\Gamma_{a b}^{p p^{\prime}}$ reads

$$
\Gamma_{a b}^{p p^{\prime}}=\delta_{a 1} \delta_{b 1} \delta_{p p^{\prime}} .
$$

Each vertex conserves the momentum and energy and it is at $O(1)$.

The chemical potential $\mu$ appears in Eqs. (A26) and (A28). Its origin is easily traced. First, the $N$-extended chemical potential term $\mu \sum_{i, p} X_{i}^{p p}$ is written as $\mu \sum_{i, p}\left(X_{i}^{p 0} X_{i}^{0 p}\right) / X_{i}^{00}$ by using the second $\delta$-function in Eq. (A3). Second, its denominator is expanded in powers of $\delta R_{i}$ [see also Eq. (A7)]. Third, to compute quantities up to $O(1 / N)$ the expansion is made up to $\delta R_{i}^{2}$, which generates a term $\mu$ in the three-leg and four-leg vertices.

The propagators and vertices are summarized in Fig. 14(a). For a large- $N$ approach, any physical quantity can be cal- culated at a given order by counting the powers of $1 / N$ in vertices and propagators involved in the corresponding Feynman diagram. In Fig. 14(a), we show the necessary diagrams to calculate quantities up to $O(1 / N)$. When going beyond that order, we need to collect more vertices in the expansion of the expression $1 /(1+\delta R)$, which demonstrates one of nontrivial aspects of the $t-J$ model.

So far, all procedures are exact formally and no approximations are introduced. Now we show calculations up to the order of $1 / N$. The bare susceptibility $D_{a b}^{(0)}$ is already at the order of $1 / N$. From the Dyson equation, the bosonic propagator (dashed line in Fig. 14) is renormalized at the same order as

$$
\left[D_{a b}\left(\mathbf{q}, i v_{n}\right)\right]^{-1}=\left[D_{a b}^{(0)}\left(\mathbf{q}, i v_{n}\right)\right]^{-1}-\Pi_{a b}\left(\mathbf{q}, i v_{n}\right),
$$

where the $6 \times 6$ boson self-energy matrix $\Pi_{a b}$ is evaluated by the diagrams shown in Fig. 14(b):

$$
\begin{aligned}
\Pi_{a b}\left(\mathbf{q}, i v_{n}\right)= & -\frac{N}{N_{s} N_{z}} \sum_{\mathbf{k}} h_{a}\left(\mathbf{k}, \mathbf{q}, \varepsilon_{\mathbf{k}}-\varepsilon_{\mathbf{k}-\mathbf{q}}\right) \\
& \times \frac{n_{F}\left(\varepsilon_{\mathbf{k}-\mathbf{q}}\right)-n_{F}\left(\varepsilon_{\mathbf{k}}\right)}{i v_{n}-\varepsilon_{\mathbf{k}}+\varepsilon_{\mathbf{k}-\mathbf{q}}} h_{b}\left(\mathbf{k}, \mathbf{q}, \varepsilon_{\mathbf{k}}-\varepsilon_{\mathbf{k}-\mathbf{q}}\right) \\
& -\delta_{a 1} \delta_{b 1} \frac{N}{N_{s} N_{z}} \sum_{\mathbf{k}} \frac{\varepsilon_{\mathbf{k}}-\varepsilon_{\mathbf{k}-\mathbf{q}}}{2} n_{F}\left(\varepsilon_{\mathbf{k}}\right) .(\mathrm{A} 34)
\end{aligned}
$$

The vertices $h_{a}$ are given by

$$
\begin{gathered}
h_{a}(\mathbf{k}, \mathbf{q}, v)=\left\{\frac{2 \varepsilon_{\mathbf{k}-\mathbf{q}}+v+2 \mu}{2}+2 \Delta\left[\cos \left(k_{x}-\frac{q_{x}}{2}\right) \cos \left(\frac{q_{x}}{2}\right)+\cos \left(k_{y}-\frac{q_{y}}{2}\right) \cos \left(\frac{q_{y}}{2}\right)\right]\right. \\
\left.1 ;-2 \Delta \cos \left(k_{x}-\frac{q_{x}}{2}\right) ;-2 \Delta \cos \left(k_{y}-\frac{q_{y}}{2}\right) ; 2 \Delta \sin \left(k_{x}-\frac{q_{x}}{2}\right) ; 2 \Delta \sin \left(k_{y}-\frac{q_{y}}{2}\right)\right\}
\end{gathered}
$$

Here the dependence on $k_{z}$ and $q_{z}$ enters only through $\epsilon_{\mathbf{k}-\mathbf{q}}$ in the first column in Eq. (A35), whereas the other columns contain the in-plane momentum only. It is important to note that the only role played by the ghost fields is to cancel infinities coming from the frequency dependence of the vertices involved in the first two diagrams in Fig. 14(b).

The Green's function acquires dynamical corrections as shown in Fig. 14(c). The self-energy consists of two parts at the order of $1 / N$ :

$$
\Sigma\left(\mathbf{k}, i \omega_{n}\right)=\Sigma^{(1)}\left(\mathbf{k}, i \omega_{n}\right)+\Sigma^{(2)}\left(\mathbf{k}, i \omega_{n}\right),
$$

where

$$
\begin{aligned}
\Sigma^{(1)}\left(\mathbf{k}, i \omega_{n}\right) & =\frac{1}{N_{s} N_{z}} \sum_{\mathbf{q}, v_{n}} \sum_{a b} \Lambda_{a}^{p p} D_{a b}\left(\mathbf{q}, i v_{n}\right) \\
& \times G_{p p}^{(0)}\left(\mathbf{k}-\mathbf{q}, i \omega_{n}-i v_{n}\right) \Lambda_{b}^{p p}
\end{aligned}
$$

and

$$
\Sigma^{(2)}\left(\mathbf{k}, i \omega_{n}\right)=\frac{1}{N_{s} N_{z}} \sum_{\mathbf{q}, v_{n}} \sum_{a b} \Lambda_{b a}^{p p} D_{a b}\left(\mathbf{q}, i v_{n}\right) .
$$

In Eqs. (A36)-(A38), we have omitted the index $p$ in the self-energy for simplicity. $\Sigma^{(1)}\left(\mathbf{k}, i \omega_{n}\right)$ corresponds to the Fock diagram, the first one in Fig. $14(\mathrm{c})$, and $\Sigma^{(2)}\left(\mathbf{k}, i \omega_{n}\right)$ the Hartree diagram, the second one in Fig. 14(c). Usually the Hartree term gives a constant contribution, which can be absorbed in the chemical potential, but here both diagrams must be considered because of the frequency and momentum dependence of the vertices $\Lambda_{a}^{p p}$ and $\Lambda_{a b}^{p p}$. One can easily see in Eqs. (A37) and (A38) that the obtained self-energy is indeed at the order of $1 / N: G_{p p}^{(0)}$ and the vertices are at $O(1)$, and $D_{a b}$ is at $O(1 / N)$.

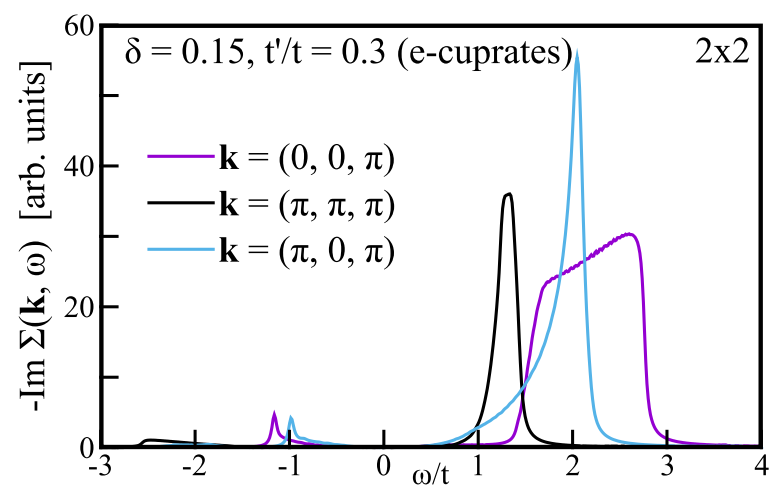

FIG. 15. Imaginary part of the electron self-energy, $-\operatorname{Im} \Sigma(\mathbf{k}, \omega)$, as a function of $\omega$ for several choices of $\mathbf{k}$ after the particle-hole transformation of Fig. 3. 
Using the spectral representation of $D_{a b}$, we obtain

$$
\begin{aligned}
\Sigma^{(1)}\left(\mathbf{k}, i \omega_{n}\right) & =-\frac{1}{\pi N_{s} N_{z}} \int d \nu \sum_{\mathbf{q}, v_{n}} \sum_{a b} \Lambda_{a}^{p p} \frac{\operatorname{Im} D_{a b}(\mathbf{q}, v)}{i v_{n}-v} \Lambda_{b}^{p p} \\
& \times G_{p p}^{(0)}\left(\mathbf{k}-\mathbf{q}, i \omega_{n}-i v_{n}\right), \\
\Sigma^{(2)}\left(\mathbf{k}, i \omega_{n}\right) & =-\frac{1}{\pi N_{s} N_{z}} \int d v \sum_{\mathbf{q}, v_{n}} \sum_{a b} \Lambda_{b a}^{p p} \frac{\operatorname{Im} D_{a b}(\mathbf{q}, v)}{i v_{n}-v} .
\end{aligned}
$$

After performing the Matsubara sum and the analytical continuation, the imaginary part of $\Sigma$ can be written in a compact
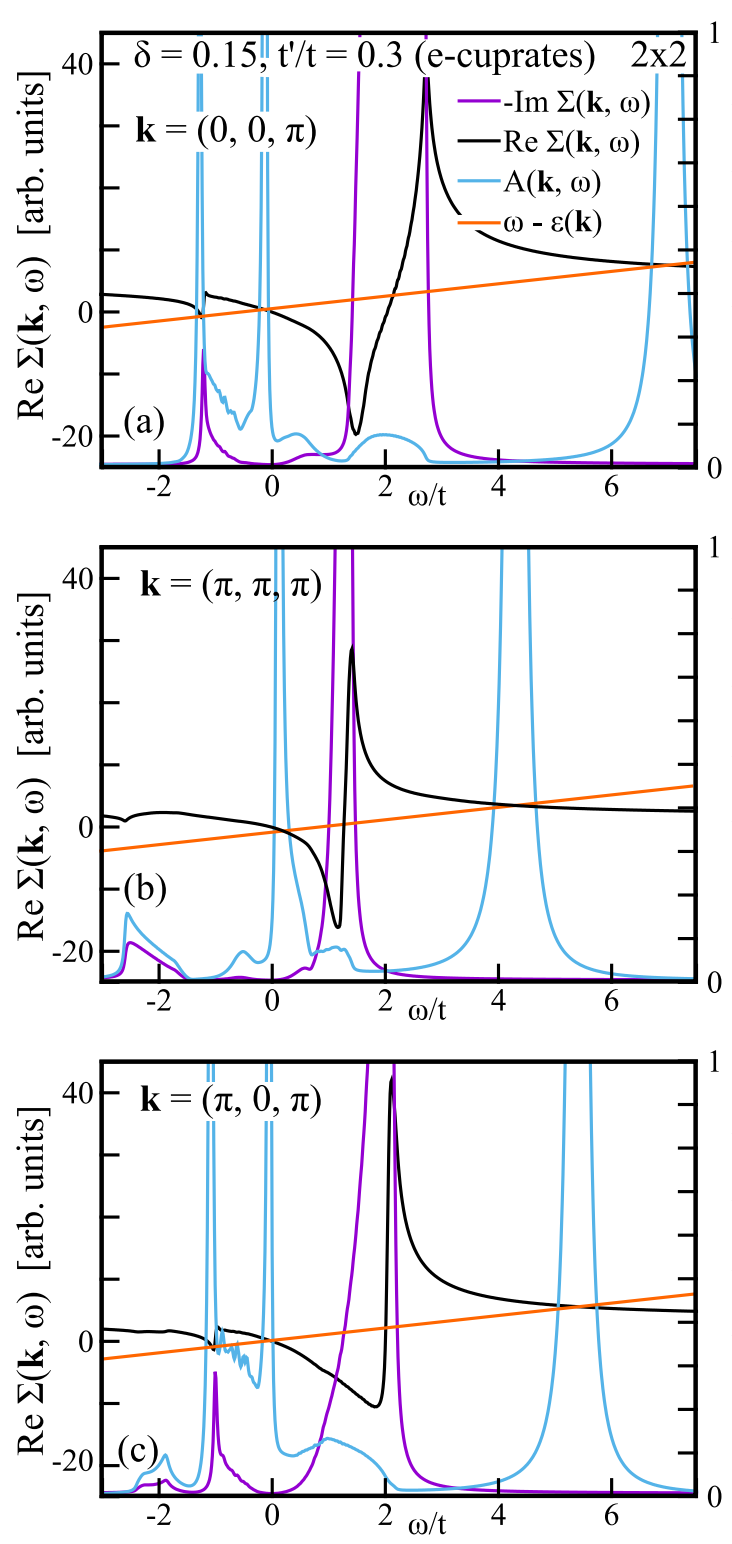

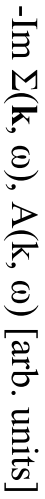

FIG. 16. Energy dependence of $-\operatorname{Im} \Sigma(\mathbf{k}, \omega), \operatorname{Re} \Sigma(\mathbf{k}, \omega)$, and $A(\mathbf{k}, \omega)$ at $\mathbf{k}=(0,0, \pi)(\mathrm{a}),(\pi, \pi, \pi)(\mathrm{b})$, and $(\pi, 0, \pi)$ (c) after the particle-hole transformation of Fig. 4. form,

$$
\begin{aligned}
\operatorname{Im} \Sigma(\mathbf{k}, \omega)= & -\frac{1}{N_{s} N_{z}} \sum_{\mathbf{q}} \sum_{a b} h_{a}\left(\mathbf{k}, \mathbf{q}, \omega-\varepsilon_{\mathbf{k}-\mathbf{q}}\right) \\
& \times \operatorname{Im} D_{a b}\left(\mathbf{q}, \omega-\varepsilon_{\mathbf{k}-\mathbf{q}}\right) h_{b}\left(\mathbf{k}, \mathbf{q}, \omega-\varepsilon_{\mathbf{k}-\mathbf{q}}\right) \\
& \times\left[n_{F}\left(-\varepsilon_{\mathbf{k}-\mathbf{q}}\right)+n_{B}\left(\omega-\varepsilon_{\mathbf{k}-\mathbf{q}}\right)\right],
\end{aligned}
$$

where $n_{B}$ is the Bose factor. We obtain $\operatorname{Re} \Sigma(\mathbf{k}, \omega)$ from $\operatorname{Im} \Sigma(\mathbf{k}, \omega)$ via the Kramers-Kronig relations. Since the electron Green's function $G(\mathbf{k}, \omega)$ is given by

$$
G(\mathbf{k}, \omega)^{-1}=\omega+i \eta-\varepsilon_{\mathbf{k}}-\Sigma(\mathbf{k}, \omega),
$$

with $\eta(>0)$ being infinitesimally small, the spectral function $A(\mathbf{k}, \omega)=-\frac{1}{\pi} \operatorname{Im} G(\mathbf{k}, \omega)$ is obtained as

$$
A(\mathbf{k}, \omega)=-\frac{1}{\pi} \frac{\operatorname{Im} \Sigma(\mathbf{k}, \omega)-\eta}{\left[\omega-\varepsilon_{\mathbf{k}}-\operatorname{Re} \Sigma(\mathbf{k}, \omega)\right]^{2}+[-\operatorname{Im} \Sigma(\mathbf{k}, \omega)+\eta]^{2}} .
$$

\section{APPENDIX B: ADDITIONAL RESULTS AFTER PARTICLE-HOLE TRANSFORMATION}

In Sec. III F, we presented results after the particle-hole transformation so they can be compared directly with experiments for e-cuprates. It is also informative to present the $\omega$ dependence of the self-energy and the spectral function. Hence we perform the particle-hole transformation of Figs. 3 and 4 and present here the corresponding results in Figs. 15 and 16 , respectively.

We also present the corresponding result to Fig. 13 in Fig. 17. This result together with Fig. 12(c) will be useful when one wishes to discuss the role of the long-range Coulomb interaction on the basis of the one-particle excitation spectrum for e-cuprates.

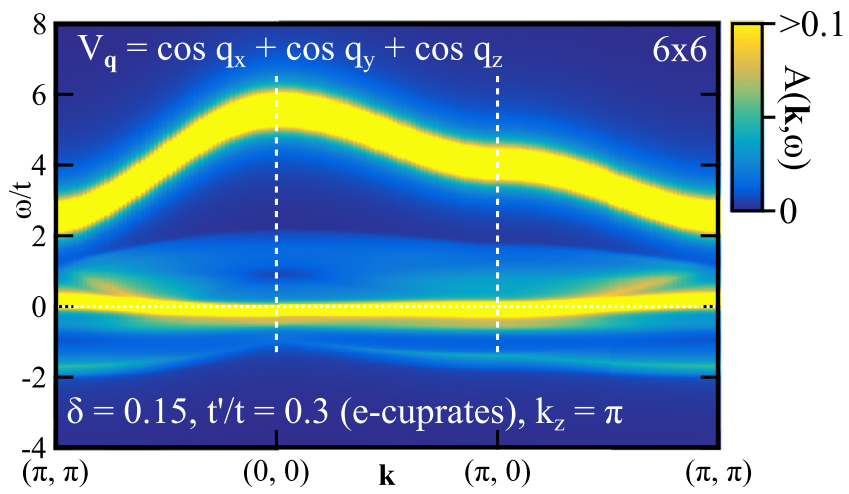

FIG. 17. Intensity map of $A(\mathbf{k}, \omega)$ in the absence of the longrange Coulomb interaction along the direction $(\pi, \pi)-(0,0)-(\pi, 0)$ $(\pi, \pi)$ in the particle picture; both on-site charge and bond-charge fluctuations are taken into account. $k_{z}$ dependence is weak and $k_{z}=$ $\pi$ is taken as a representative value. The corresponding result in the hole picture is presented in Fig. 13. 
[1] B. Keimer, S. A. Kivelson, M. R. Norman, S. Uchida, and J. Zaanen, From quantum matter to high-temperature superconductivity in copper oxides, Nature (London) 518, 179 (2015).

[2] K. Ishii, K. Tsutsui, Y. Endoh, T. Tohyama, S. Maekawa, M. Hoesch, K. Kuzushita, M. Tsubota, T. Inami, J. Mizuki, Y. Murakami, and K. Yamada, Momentum Dependence of Charge Excitations in the Electron-Doped Superconductor $\mathrm{Nd}_{1.85} \mathrm{Ce}_{0.15} \mathrm{CuO}_{4}$ : A Resonant Inelastic X-Ray Scattering Study, Phys. Rev. Lett. 94, 207003 (2005).

[3] G. Ghiringhelli, M. Le Tacon, M. Minola, S. Blanco-Canosa, C. Mazzoli, N. B. Brookes, G. M. De Luca, A. Frano, D. G. Hawthorn, F. He, T. Loew, M. M. Sala, D. C. Peets, M. Salluzzo, E. Schierle, R. Sutarto, G. A. Sawatzky, E. Weschke, B. Keimer, and L. Braicovich, Long-range incommensurate charge fluctuations in (Y, Nd) $\mathrm{Ba}_{2} \mathrm{Cu}_{3} \mathrm{O}_{6+x}$, Science 337, 821 (2012).

[4] J. Chang, E. Blackburn, A. T. Holmes, N. B. Christensen, J. Larsen, J. Mesot, R. Liang, D. A. Bonn, W. N. Hardy, A. Watenphul, M. v. Zimmermann, E. M. Forgan, and S. M. Hayden, Direct observation of competition between superconductivity and charge density wave order in $\mathrm{YBa}_{2} \mathrm{Cu}_{3} \mathrm{O}_{6.67}$, Nat. Phys. 8, 871 (2012).

[5] A. J. Achkar, R. Sutarto, X. Mao, F. He, A. Frano, S. Blanco-Canosa, M. Le Tacon, G. Ghiringhelli, L. Braicovich, M. Minola, M. Moretti Sala, C. Mazzoli, R. Liang, D. A. Bonn, W. N. Hardy, B. Keimer, G. A. Sawatzky, and D. G. Hawthorn, Distinct Charge Orders in the Planes and Chains of Ortho-III-Ordered $\mathrm{YBa}_{2} \mathrm{Cu}_{3} \mathrm{O}_{6+\delta}$ Superconductors Identified by Resonant Elastic X-Ray Scattering, Phys. Rev. Lett. 109, 167001 (2012).

[6] E. H. da Silva Neto, R. Comin, F. He, R. Sutarto, Y. Jiang, R. L. Greene, G. A. Sawatzky, and A. Damascelli, Charge ordering in the electron-doped superconductor $\mathrm{Nd}_{2-x} \mathrm{Ce}_{x} \mathrm{CuO}_{4}$, Science 347, 282 (2015).

[7] W. S. Lee, J. J. Lee, E. A. Nowadnick, S. Gerber, W. Tabis, S. W. Huang, V. N. Strocov, E. M. Motoyama, G. Yu, B. Moritz, H. Y. Huang, R. P. Wang, Y. B. Huang, W. B. Wu, C. T. Chen, D. J. Huang, M. Greven, T. Schmitt, Z. X. Shen, and T. P. Devereaux, Asymmetry of collective excitations in electron- and hole-doped cuprate superconductors, Nat. Phys. 10, 883 (2014).

[8] K. Ishii, M. Fujita, T. Sasaki, M. Minola, G. Dellea, C. Mazzoli, K. Kummer, G. Ghiringhelli, L. Braicovich, T. Tohyama, K. Tsutsumi, K. Sato, R. Kajimoto, K. Ikeuchi, K. Yamada, M. Yoshida, M. Kurooka, and J. Mizuki, High-energy spin and charge excitations in electron-doped copper oxide superconductors, Nat. Commun. 5, 3714 (2014).

[9] E. H. da Silva Neto, B. Yu, M. Minola, R. Sutarto, E. Schierle, F. Boschini, M. Zonno, M. Bluschke, J. Higgins, Y. Li, G. Yu, E. Weschke, F. He, M. Le Tacon, R. L. Greene, M. Greven, G. A. Sawatzky, B. Keimer, and A. Damascelli, Doping-dependent charge order correlations in electron-doped cuprates, Sci. Adv. 2, e1600782 (2016).

[10] G. Dellea, M. Minola, A. Galdi, D. Di Castro, C. Aruta, N. B. Brookes, C. J. Jia, C. Mazzoli, M. Moretti Sala, B. Moritz, P. Orgiani, D. G. Schlom, A. Tebano, G. Balestrino, L. Braicovich, T. P. Devereaux, L. Maritato, and G. Ghiringhelli, Spin and charge excitations in artificial hole- and electrondoped infinite layer cuprate superconductors, Phys. Rev. B 96, 115117 (2017).
[11] M. Hepting, L. Chaix, E. W. Huang, R. Fumagalli, Y. Y. Peng, B. Moritz, K. Kummer, N. B. Brookes, W. C. Lee, M. Hashimoto, T. Sarkar, J.-F. He, C. R. Rotundu, Y. S. Lee, R. L. Greene, L. Braicovich, G. Ghiringhelli, Z. X. Shen, T. P. Devereaux, and W. S. Lee, Three-dimensional collective charge excitations in electron-doped copper oxide superconductors, Nature (London) 563, 374 (2018).

[12] J. Lin, J. Yuan, K. Jin, Z. Yin, G. Li, K.-J. Zhou, X. Lu, M. Dantz, T. Schmitt, H. Ding, H. Guo, M. P. M. Dean, and X. Liu, Doping evolution of the charge excitations and electron correlations in electron-doped superconducting $\mathrm{La}_{2-\mathrm{x}} \mathrm{Ce}_{\mathrm{x}} \mathrm{CuO}_{4}$, npj Quantum Mater. 5, 4 (2020).

[13] M. Bejas, H. Yamase, and A. Greco, Dual structure in the charge excitation spectrum of electron-doped cuprates, Phys. Rev. B 96, 214513 (2017).

[14] H. Yamase, M. Bejas, and A. Greco, d-wave bond-order charge excitations in electron-doped cuprates, Europhys. Lett. 111, 57005 (2015).

[15] H. Yamase, M. Bejas, and A. Greco, Doping dependence of $d$-wave bond-charge excitations in electron-doped cuprates, Phys. Rev. B 99, 014513 (2019).

[16] A. Greco, H. Yamase, and M. Bejas, Plasmon excitations in layered high- $T_{c}$ cuprates, Phys. Rev. B 94, 075139 (2016).

[17] A. Greco, H. Yamase, and M. Bejas, Origin of high-energy charge excitations observed by resonant inelastic x-ray scattering in cuprate superconductors, Commun. Phys. 2, 3 (2019).

[18] A. Greco, H. Yamase, and M. Bejas, Close inspection of plasmon excitations in cuprate superconductors, Phys. Rev. B 102, 024509 (2020).

[19] M. Bejas, A. Greco, and H. Yamase, Possible charge instabilities in two-dimensional doped Mott insulators, Phys. Rev. B 86, 224509 (2012).

[20] A. Allais, J. Bauer, and S. Sachdev, Density wave instabilities in a correlated two-dimensional metal, Phys. Rev. B 90, 155114 (2014).

[21] H. Meier, C. Pépin, M. Einenkel, and K. B. Efetov, Cascade of phase transitions in the vicinity of a quantum critical point, Phys. Rev. B 89, 195115 (2014).

[22] Y. Wang and A. Chubukov, Charge-density-wave order with momentum $(2 q, 0)$ and $(0,2 q)$ within the spin-fermion model: Continuous and discrete symmetry breaking, preemptive composite order, and relation to pseudogap in hole-doped cuprates, Phys. Rev. B 90, 035149 (2014).

[23] W. A. Atkinson, A. P. Kampf, and S. Bulut, Charge order in the pseudogap phase of cuprate superconductors, New J. Phys. 17, 013025 (2015).

[24] Y. Yamakawa and H. Kontani, Spin-Fluctuation-Driven Nematic Charge-Density Wave in Cuprate Superconductors: Impact of Aslamazov-Larkin Vertex Corrections, Phys. Rev. Lett. 114, 257001 (2015).

[25] V. Mishra and M. R. Norman, Strong coupling critique of spin fluctuation driven charge order in underdoped cuprates, Phys. Rev. B 92, 060507(R) (2015).

[26] R. Zeyher and A. Greco, Competition between spin-induced charge instabilities in underdoped cuprates, Phys. Rev. B 98, 224504 (2018).

[27] K. Ishii, T. Tohyama, S. Asano, K. Sato, M. Fujita, S. Wakimoto, K. Tustsui, S. Sota, J. Miyawaki, H. Niwa, Y. Harada, J. Pelliciari, Y. Huang, T. Schmitt, Y. Yamamoto, and J. Mizuki, Observation of momentum-dependent charge exci- 
tations in hole-doped cuprates using resonant inelastic x-ray scattering at the oxygen $K$ edge, Phys. Rev. B 96, 115148 (2017).

[28] A. Singh, H. Y. Huang, C. Lane, J. H. Li, J. Okamoto, S. Komiya, R. S. Markiewicz, A. Bansil, A. Fujimori, C. T. Chen, and D. J. Huang, arXiv:2006.13424.

[29] A. Nag, M. Zhu, M. Bejas, J. Li, H. C. Robarts, H. Yamase, A. N. Petsch, D. Song, H. Eisaki, A. C. Walters, M. GarcíaFernández, A. Greco, S. M. Hayden, and K.-J. Zhou, Detection of Acoustic Plasmons in Hole-Doped Lanthanum and Bismuth Cuprate Superconductors using Resonant Inelastic x-ray Scattering, Phys. Rev. Lett. 125, 257002 (2020).

[30] M. Mitrano, A. A. Husain, S. Vig, A. Kogar, M. S. Rak, S. I. Rubeck, J. Schmalian, B. Uchoa, J. Schneeloch, R. Zhong, G. D. Gu, and P. Abbamonte, Anomalous density fluctuations in a strange metal, Proc. Natl. Acad. Sci. USA 115, 5392 (2018).

[31] A. A. Husain, M. Mitrano, M. S. Rak, S. Rubeck, B. Uchoa, K. March, C. Dwyer, J. Schneeloch, R. Zhong, G. D. Gu, and P. Abbamonte, Crossover of Charge Fluctuations Across the Strange Metal Phase Diagram, Phys. Rev. X 9, 041062 (2019).

[32] R. S. Markiewicz and A. Bansil, Dispersion anomalies induced by the low-energy plasmon in the cuprates, Phys. Rev. B 75, 020508(R) (2007).

[33] M. M. Zemljič, P. Prelovšek, and T. Tohyama, Temperature and Doping Dependence of the High-Energy Kink in Cuprates, Phys. Rev. Lett. 100, 036402 (2008).

[34] J. Graf, G.-H. Gweon, K. McElroy, S. Y. Zhou, C. Jozwiak, E. Rotenberg, A. Bill, T. Sasagawa, H. Eisaki, S. Uchida, H. Takagi, D.-H. Lee, and A. Lanzara, Universal High Energy Anomaly in the Angle-Resolved Photoemission Spectra of High Temperature Superconductors: Possible Evidence of Spinon and Holon Branches, Phys. Rev. Lett. 98, 067004 (2007).

[35] B. P. Xie, K. Yang, D. W. Shen, J. F. Zhao, H. W. Ou, J. Weil, S. Y. Gu, M. Arita, S. Qiao, H. Namatame, M. Taniguchi, N. Kaneko, H. Eisaki, K. D. Tsuei, C. M. Cheng, I. Vobornik, J. Fujii, G. Rossi, Z. Q. Yang, and D. L. Feng, High-Energy Scale Revival and Giant Kink in the Dispersion of a Cuprate Superconductor, Phys. Rev. Lett. 98, 147001 (2007).

[36] T. Valla, T. E. Kidd, W.-G. Yin, G. D. Gu, P. D. Johnson, Z.-H. Pan, and A. V. Fedorov, High-Energy Kink Observed in the Electron Dispersion of High-Temperature Cuprate Superconductors, Phys. Rev. Lett. 98, 167003 (2007).

[37] W. Meevasana, X. J. Zhou, S. Sahrakorpi, W. S. Lee, W. L. Yang, K. Tanaka, N. Mannella, T. Yoshida, D. H. Lu, Y. L. Chen, R. H. He, H. Lin, S. Komiya, Y. Ando, F. Zhou, W. X. Ti, J. W. Xiong, Z. X. Zhao, T. Sasagawa, T. Kakeshita et al., Hierarchy of multiple many-body interaction scales in high-temperature superconductors, Phys. Rev. B 75, 174506 (2007).

[38] J. Chang, S. Pailhés, M. Shi, M. Månson, T. Claesson, O. Tjernberg, J. Voigt, V. Perez, L. Patthey, N. Momono, M. Oda, M. Ido, A. Schnyder, C. Mudry, and J. Mesot, When low- and high-energy electronic responses meet in cuprate superconductors, Phys. Rev. B 75, 224508 (2007).

[39] W. Zhang, G. Liu, J. Meng, L. Zhao, H. Liu, X. Dong, W. Lu, J. S. Wen, Z. J. Xu, G. D. Gu, T. Sasagawa, G. Wang, Y. Zhu, H. Zhang, Y. Zhou, X. Wang, Z. Zhao,
C. Chen, Z. Xu, and X. J. Zhou, High Energy Dispersion Relations for the High Temperature $\mathrm{Bi}_{2} \mathrm{Sr}_{2} \mathrm{CaCu}_{2} \mathrm{O}_{8}$ Superconductor from Laser-Based Angle-Resolved Photoemission Spectroscopy, Phys. Rev. Lett. 101, 017002 (2008).

[40] T. Thio, T. R. Thurston, N. W. Preyer, P. J. Picone, M. A. Kastner, H. P. Jenssen, D. R. Gabbe, C. Y. Chen, R. J. Birgeneau, and A. Aharony, Antisymmetric exchange and its influence on the magnetic structure and conductivity of $\mathrm{La}_{2} \mathrm{CuO}_{4}$, Phys. Rev. B 38, 905 (1988).

[41] D. Grecu, Plasma frequency of the electron gas in layered structures, Phys. Rev. B 8, 1958 (1973).

[42] A. L. Fetter, Electrodynamics of a layered electron gas. II. Periodic array, Ann. Phys. 88, 1 (1974).

[43] D. Grecu, Self-consistent field approximation for the plasma frequencies of an electron gas in a layered thin film, J. Phys. C 8, 2627 (1975).

[44] A. Foussats and A. Greco, Large- $N$ expansion based on the Hubbard operator path integral representation and its application to the $t$ - $J$ model, Phys. Rev. B 65, 195107 (2002).

[45] M. Bejas, A. Greco, and H. Yamase, Strong particle-hole asymmetry of charge instabilities in doped Mott insulators, New J. Phys. 16, 123002 (2014).

[46] Z. Wang, Y. Bang, and G. Kotliar, Collective Excitations and Spectral Function in the Fermi-Liquid State of the $t$ - $J$ Model, Phys. Rev. Lett. 67, 2733 (1991).

[47] J. Merino, A. Greco, R. H. McKenzie, and M. Calandra, Dynamical properties of a strongly correlated model for quarter-filled layered organic molecular crystals, Phys. Rev. B 68, 245121 (2003).

[48] M. Bejas, A. Greco, and A. Foussats, One-electron selfenergies and spectral functions for the $t-J$ model in the large- $N$ limit, Phys. Rev. B 73, 245104 (2006).

[49] F. Becca, M. Tarquini, M. Grilli, and C. Di Castro, Chargedensity waves and superconductivity as an alternative to phase separation in the infinite- $U$ Hubbard-Holstein model, Phys. Rev. B 54, 12443 (1996).

[50] F. C. Zhang and T. M. Rice, Effective Hamiltonian for the superconducting Cu oxides, Phys. Rev. B 37, 3759 (1988).

[51] Z. Wang, Strongly correlated Fermi liquid and the normal state of high $\mathrm{T}_{\mathrm{c}}$ superconductors, Int. J. Mod. Phys. B 6, 603 (1992).

[52] G. Kotliar and J. Liu, Superconducting Instabilities in the Large- $U$ Limit of a Generalized Hubbard Model, Phys. Rev. Lett. 61, 1784 (1988).

[53] D. C. Morse and T. C. Lubensky, Instabilities of the Fermiliquid and staggered flux phases in the large- $N t$ - $J$ model, Phys. Rev. B 43, 10436 (1991).

[54] P. Prelovšek and P. Horsch, Electron-energy loss spectra and plasmon resonance in cuprates, Phys. Rev. B 60, R3735 (1999).

[55] A. Foussats, A. Greco, and M. Bejas, Role of dynamical nondouble-occupancy excitations on the quasiparticle damping of the $t-J$ model in the large- $N$ limit, Phys. Rev. B 78, 153110 (2008).

[56] A. Greco, H. Yamase, and M. Bejas, Charge-density-excitation spectrum in the $t-t^{\prime}-J-V$ model, J. Phys. Soc. Jpn. 86, 034706 (2017).

[57] H. Yamase and H. Kohno, Possible quasi-one-dimensional Fermi surface in $\mathrm{La}_{2-x} \mathrm{Sr}_{x} \mathrm{CuO}_{4}$, J. Phys. Soc. Jpn. 69, 332 (2000). 
[58] H. Yamase and H. Kohno, Instability toward formation of quasi-one-dimensional Fermi surface in two-dimensional $t$ - $J$ model, J. Phys. Soc. Jpn. 69, 2151 (2000).

[59] C. J. Halboth and W. Metzner, $d$-Wave Superconductivity and Pomeranchuk Instability in the Two-Dimensional Hubbard Model, Phys. Rev. Lett. 85, 5162 (2000).

[60] W. Metzner, D. Rohe, and S. Andergassen, Soft Fermi Surfaces and Breakdown of Fermi-Liquid Behavior, Phys. Rev. Lett. 91, 066402 (2003).

[61] H. Yamase, Excitation Spectrum of $d$-Wave Fermi Surface Deformation, Phys. Rev. Lett. 93, 266404 (2004).

[62] M. A. Metlitski and S. Sachdev, Quantum phase transitions of metals in two spatial dimensions. I. Ising-nematic order, Phys. Rev. B 82, 075127 (2010).

[63] T. Holder and W. Metzner, Incommensurate nematic fluctuations in two-dimensional metals, Phys. Rev. B 85, 165130 (2012).

[64] I. Affleck and J. B. Marston, Large-n limit of the HeisenbergHubbard model: Implications for high- $T_{c}$ superconductors, Phys. Rev. B 37, 3774 (1988).

[65] E. Cappelluti and R. Zeyher, Interplay between superconductivity and flux phase in the $t-J$ model, Phys. Rev. B 59, 6475 (1999).

[66] S. Chakravarty, R. B. Laughlin, D. K. Morr, and C. Nayak, Hidden order in the cuprates, Phys. Rev. B 63, 094503 (2001).

[67] It is possible to define a particle-hole transformation with $\mathbf{Q}=$ $(\pi, \pi, \pi)$. Even in this case, our conclusions are not modified.

[68] A. Greco and R. Zeyher, Influence of spin fluctuations on the superconducting transition temperature and resistivity in the $t$ - $J$ model at large N, Phys. Rev. B 63, 064520 (2001).

[69] N. M. Plakida and V. S. Oudovenko, Kinematic spinfluctuation mechanism of high-temperature superconductivity, J. Exp. Theor. Phys. 119, 554 (2014).

[70] P. Prelovšek and A. Ramšak, Spectral functions and the pseudogap in the $t-J$ model, Phys. Rev. B 63, 180506(R) (2001).

[71] W. Drube, F. J. Himpsel, G. V. Chandrashekhar, and M. W. Shafer, Empty states near the Fermi level in $\mathrm{Bi}_{2} \mathrm{Sr}_{2} \mathrm{CaCu}_{2} \mathrm{O}_{8}$, Phys. Rev. B 39, 7328 (1989).

[72] T. J. Wagener, Y. Hu, Y. Gao, M. B. Jost, J. H. Weaver, N. D. Spencer, and K. C. Goretta, Resonant inverse photoemission of $\mathrm{Bi}_{2} \mathrm{Ca}_{1+x} \mathrm{Sr}_{2-x} \mathrm{Cu}_{2} \mathrm{O}_{8+y}$ and $\mathrm{YBa}_{2} \mathrm{Cu}_{3} \mathrm{O}_{7-x}$, unoccupied oxygen states, and plasmons, Phys. Rev. B 39, 2928 (1989).

[73] A factor of $1 / 2$ here comes from a large- $N$ formalism where $t$ is scaled by $1 / N$. We may assume $N=2$ in comparison with experiments.

[74] M. S. Hybertsen, E. B. Stechel, M. Schluter, and D. R. Jennison, Renormalization from density-functional theory to strong-coupling models for electronic states in $\mathrm{Cu}-\mathrm{O}$ materials, Phys. Rev. B 41, 11068 (1990).

[75] F. Tan and Q.-H. Wang, Two-Mode Variational Monte Carlo Study of Quasiparticle Excitations in Cuprate Superconductors, Phys. Rev. Lett. 100, 117004 (2008).

[76] C. Weber, K. Haule, and G. Kotliar, Apical oxygens and correlation strength in electron- and hole-doped copper oxides, Phys. Rev. B 82, 125107 (2010).

[77] N. P. Armitage, P. Fournier, and R. L. Greene, Progress and perspectives on electron-doped cuprates, Rev. Mod. Phys. 82, 2421 (2010)
[78] A. Greco, Evidence for Two Competing Order Parameters in Underdoped Cuprate Superconductors from a Model Analysis of Fermi-Arc Effects, Phys. Rev. Lett. 103, 217001 (2009).

[79] H. Yamase and W. Metzner, Fermi-Surface Truncation from Thermal Nematic Fluctuations, Phys. Rev. Lett. 108, 186405 (2012).

[80] A. Kaminski, M. Randeria, J. C. Campuzano, M. R. Norman, H. Fretwell, J. Mesot, T. Sato, T. Takahashi, and K. Kadowaki, Renormalization of Spectral Line Shape and Dispersion Below $T_{c}$ in $\mathrm{Bi}_{2} \mathrm{Sr}_{2} \mathrm{CaCu}_{2} \mathrm{O}_{8+\delta}$, Phys. Rev. Lett. 86, 1070 (2001).

[81] A. Lanzara, P. V. Bogdanov, X. J. Zhou, S. A. Kellar, D. L. Feng, E. D. Lu, T. Yoshida, H. Eisaki, A. Fujimori, K. Kishio, J.-I. Shimoyama, T. Noda, S. Uchida, Z. Hussain, and Z.-X. Shen, Evidence for ubiquitous strong electron-phonon coupling in high-temperature superconductors, Nature (London) 412, 510 (2001).

[82] P. D. Johnson, T. Valla, A. V. Fedorov, Z. Yusof, B. O. Wells, Q. Li, A. R. Moodenbaugh, G. D. Gu, N. Koshizuka, C. Kendziora, S. Jian, and D. G. Hinks, Doping and Temperature Dependence of the Mass Enhancement Observed in the Cuprate $\mathrm{Bi}_{2} \mathrm{Sr}_{2} \mathrm{CaCu}_{2} \mathrm{O}_{8+\delta}$, Phys. Rev. Lett. 87, 177007 (2001).

[83] A. D. Gromko, A. V. Fedorov, Y.-D. Chuang, J. D. Koralek, Y. Aiura, Y. Yamaguchi, K. Oka, Y. Ando, and D. S. Dessau, Mass-renormalized electronic excitations at $(\pi, 0)$ in the superconducting state of $\mathrm{Bi}_{2} \mathrm{Sr}_{2} \mathrm{CaCu}_{2} \mathrm{O}_{8+\delta}$, Phys. Rev. B 68, 174520 (2003).

[84] X. J. Zhou, J. Shi, T. Yoshida, T. Cuk, W. L. Yang, V. Brouet, J. Nakamura, N. Mannella, S. Komiya, Y. Ando, F. Zhou, W. X. Ti, J. W. Xiong, Z. X. Zhao, T. Sasagawa, T. Kakeshita, H. Eisaki, S. Uchida, A. Fujimori, Z. Zhang et al., Multiple Bosonic Mode Coupling in the Electron Self-Energy of $\left(\mathrm{La}_{2-x} \mathrm{Sr}_{x}\right) \mathrm{CuO}_{4}$, Phys. Rev. Lett. 95, 117001 (2005).

[85] D. Mou, A. Kaminski, and G. Gu, Direct observation of self-energy signatures of the resonant collective mode in $\mathrm{Bi}_{2} \mathrm{Sr}_{2} \mathrm{CaCu}_{2} \mathrm{O}_{8+\delta}$, Phys. Rev. B 95, 174501 (2017).

[86] T. Valla, I. K. Drozdov, and G. D. Gu, Disappearance of superconductivity due to vanishing coupling in the overdoped $\mathrm{Bi}_{2} \mathrm{Sr}_{2} \mathrm{CaCu}_{2} \mathrm{O}_{8+\delta}$, Nat. Commun. 11, 569 (2020).

[87] M. Eschrig and M. R. Norman, Neutron Resonance: Modeling Photoemission and Tunneling Data in the Superconducting State of $\mathrm{Bi}_{2} \mathrm{Sr}_{2} \mathrm{CaCu}_{2} \mathrm{O}_{8+\delta}$, Phys. Rev. Lett. 85, 3261 (2000).

[88] J. P. Carbotte, T. Timusk, and J. Hwang, Bosons in hightemperature superconductors: An experimental survey, Rep. Prog. Phys. 74, 066501 (2011).

[89] R. S. Markiewicz, S. Sahrakorpi, and A. Bansil, Paramagnoninduced dispersion anomalies in the cuprates, Phys. Rev. B 76, 174514 (2007).

[90] R. Zeyher and A. Greco, Low-energy renormalization of the electron dispersion of high- $T_{c}$ superconductors, Phys. Rev. B 64, 140510(R) (2001).

[91] K. Byczuk, M. Kollar, K. Held, Y. F. Yang, I. A. Nekrasov, T. Pruschke, and D. Vollhardt, Kinks in the dispersion of strongly correlated electrons, Nat. Phys. 3, 168 (2007).

[92] A. Macridin, M. Jarrell, T. Maier, and D. J. Scalapino, High-Energy Kink in the Single-Particle Spectra of the TwoDimensional Hubbard Model, Phys. Rev. Lett. 99, 237001 (2007). 
[93] S. Kar and E. Manousakis, Hole spectral functions in lightly doped quantum antiferromagnets, Phys. Rev. B 84, 205107 (2011).

[94] E. Manousakis, String excitations of a hole in a quantum antiferromagnet and photoelectron spectroscopy, Phys. Rev. B 75, 035106 (2007).

[95] D. S. Inosov, J. Fink, A. A. Kordyuk, S. V. Borisenko, V. B. Zabolotnyy, R. Schuster, M. Knupfer, B. Büchner, R. Follath, H. A. Dürr, W. Eberhardt, V. Hinkov, B. Keimer, and $\mathrm{H}$. Berger, Momentum and Energy Dependence of the Anomalous High-Energy Dispersion in the Electronic Structure of High Temperature Superconductors, Phys. Rev. Lett. 99, 237002 (2007).

[96] M. Fleck, A. I. Lichtenstein, and A. M. Oleś, Spectral properties and pseudogap in the stripe phases of cuprate superconductors, Phys. Rev. B 64, 134528 (2001).

[97] J. Hubbard, Electron correlations in narrow energy bands, Proc. R. Soc. London A 276, 238 (1963).
[98] J. C. Le Guillou and E. Ragoucy, Slave-particle quantization and sum rules in the $t$ - $J$ model, Phys. Rev. B 52, 2403 (1995).

[99] L. Faddeev and R. Jackiw, Hamiltonian Reduction of Unconstrained and Constrained Systems, Phys. Rev. Lett. 60, 1692 (1988).

[100] P. A. M. Dirac, Lectures on Quantum Mechanics (Yeshiva University Press, New York, 1964).

[101] K. Sundermeyer, Constrained Dynamics (Springer, Berlin, 1982).

[102] A. Foussats, A. Greco, C. Repetto, O. P. Zandron, and O. S. Zandron, Connection between the slave-particle and $\mathrm{x}$-operator path-integral representations. A new perturbative approach, J. Phys. A: Math. Gen. 33, 5849 (2000).

[103] A. Foussats, A. Greco, and O. Zandron, First-order Lagrangians and path-integral quantization in the $t$ - $J$ model, Ann. Phys. 275, 238 (1999).

[104] P. B. Wiegmann, Superconductivity in Strongly Correlated Electronic Systems and Confinement Versus Deconfinement Phenomenon, Phys. Rev. Lett. 60, 821 (1988). 